\title{
EXISTENCE THEOREMS FOR MATROID DESIGNS
}

BY

\section{H. PEYTON YOUNG $\left({ }^{1}\right)$}

ABSTRACT. A study is made of matroids in which the hyperplanes have equal cardinality. Fundamental constructions of such matroids are exhibited, and existence theorems are proved for large parametric classes of them.

Introduction. U. S. R. Murty [10] was the first to study matroids in which the hyperplanes all have the same cardinality. He called these structures "equicardinal matroids". As we shall presently show, such matroids turn out to be closely related to certain special classes of balanced incomplete block designs (BIBD's). Hence we have chosen to rechristen them matroid designs. If, in addition, one requires of a matroid that the flats of any given rank have the same cardinality, it is called a perfect matroid design. Perfect matroid designs have been studied by J. Edmonds and the present author in [15].

In [10], Murty posed the problem of finding, for each triple of integers $v, k$, and $r$, all matroid designs having $v$ points, hyperplane cardinality $k$, and rank $r$. In the present paper we develop a general framework for solving this problem, and then proceed to list all possible matroid designs for large classes of integers $v$, $k$, and $r$.

It turns out that for some such integers $v$ and $k$ the only matroid designs of any rank are trivial ones (truncations of Boolean algebras), and for other integer pairs the only possibilities are certain classes of BIBD's and alterations of BIBD's. Nevertheless, we shall present a sufficient variety of examples to indicate that the diversity and complexity possible in matroid designs is probably unlimited.

A number of the results in the present paper have previously been announced in summary fashion in [14]. The matroid concepts we will be using are developed in detail in [15]; however, for the sake of completeness we will briefly recall some of the basic definitions and theorems here. The interested reader might also

Received by the editors February 11, 1972.

AMS (MOS) subject classifications (1970). Primary 05B05, 05B25, $05 \mathrm{~B} 35$.

Key words and phrases. Matroid, block design, finite projective and affine geometries.

(1) This work was completed at the University of Waterloo, 1969-1970, and appeared as a part of the author's $\mathrm{Ph}$. D. thesis (University of Michigan, 1970), under the supervision of Jack Edmonds. The author wishes to especially thank Jack Edmonds for his encouragement and advice; also U.S. R. Murty, for contributing many examples of matroid designs; and C. St. J. A. Nash-Williams for providing financial support. 
refer to the papers of Tutte [11], Whitney [12], and Crapo and Rota [3] for general background.

(1) A matroid $M=(E, 9)$ is defined to be a finite set $E$ together with a nonempty set 9 of subsets of $E$, called independent sets, such that

(I1) Every subset of an independent set is independent.

(I2) For every $A \subseteq E$, all maximal independent subsets of $A$ have the same cardinality, called the rank of $A$, and denoted by $r(A)$.

(2) Any maximal independent subset of a set $A \subseteq E$ is called a basis of $A$. $A$ basis of $M$ is just a basis of $E$, and $r(M)$, the rank of $M$, is defined to be $r(E)$.

(3) A subset of $E$ that is not independent is said to be dependent, and the minimal dependent sets are called circuits. For any element $x \in E$ and $A \subseteq E, x$ is said to depend on $A$ if $x \in A$ or $\{x\} \cup A$ contains a circuit containing $x$. The closure of $A, \operatorname{cl}(A)$, is the set of all elements that depend on $A$, and $A$ is said to be a closed set, or flat, if $\operatorname{cl}(A)=A$. We shall write $\operatorname{cl}_{M}(A)$ if we wish to emphasize that closure is taken with respect to $M$.

(4) It follows easily from the above definition that the intersection of a family of closed sets is closed.

We may characterize the closure of a set as follows.

(5) For any $A \subseteq E, \operatorname{cl}(A)$ is the unique maximal set $S$ such that $A \subseteq S \subseteq E$ and $r(A)=r(S)$.

(6) A $k$-flat of $M$ is defined to be a closed subset of $E$ having rank $k$. The byperplanes of $M$ are its $(r(M)-1)$-flats.

(7) If $M$ is a matroid design, we will denote the common cardinality of its hyperplanes by $k(M)$.

(8) For any subset $E^{\prime}$ of $E$, let $g^{\prime}=\left\{J \subseteq E^{\prime}: J \in 9\right\} . M^{\prime}=\left(E^{\prime}, g^{\prime}\right)$ satisfies (I1) and (I2), and is called the submatroid of $M$ on $E^{\prime}$, or the reduction of $M$ to $E^{\prime}$, denoted by $M \times E^{\prime}$. Clearly, the rank function, $r^{\prime}$, of $M^{\prime}$ is just the rank function of $M$ restricted to the subsets of $E^{\prime}$.

(9) Let $E^{\prime} \subseteq E$, and let $g^{\prime}=\left\{J^{\prime} \subseteq E^{\prime}: J^{\prime} \cup J \in 9\right.$ for some M-basis $J$ of $\left.E-E^{\prime}\right\}$. It is easily verified that $\left(E^{\prime}, g^{\prime}\right)$ satisfies (I1) and (I2); hence it is a matroid, called the contraction of $M$ to $E^{\prime}$, and denoted by $M \cdot E^{\prime}$. For any $A \subseteq E^{\prime}$, the rank of $A$ in $M \cdot E^{\prime}$ is given by

$$
r^{\prime}(A)=r\left(A \cup\left(E-E^{\prime}\right)\right)-r\left(E-E^{\prime}\right),
$$

where $r$ is the rank function of $M$. In particular,

$$
r\left(M \cdot E^{\prime}\right)=r(M)-r\left(E-E^{\prime}\right) .
$$

The flats of $M \cdot E^{\prime}$ are characterized in terms of the flats of $M$ as follows. 
(12) Theorem. For $E^{\prime} \subseteq E$ and $0 \leq j \leq r(M)-r\left(E-E^{\prime}\right), F \subseteq E^{\prime}$ is a j-flat of $M \cdot E^{\prime}$ if and only if $F \cup\left(E-E^{\prime}\right)$ is a $j+r\left(E-E^{\prime}\right)$-flat of $M$.

(13) Corollary. If $M$ is a matroid design, and $E^{\prime} \subseteq E$, then $M \cdot E^{\prime}$ is a matroid design and $k\left(M \cdot E^{\prime}\right)=k(M)-\left|E-E^{\prime}\right|$.

(14) Let $F^{i} \subseteq F^{k}$ be an $i$-flat and $k$-flat respectively. The interval of $M$ between $F^{i}$ and $F^{k}, M\left(F^{i}, F^{k}\right)$, is the matroid $\left(M \times F^{k}\right) \cdot\left(F^{k}-F^{i}\right)$.

(15) It is easily seen that the flats of an interval $M\left(F^{i}, F^{k}\right)$ of $M$ correspond 1-1 with the flats $A$ of $M$ such that $F^{i} \subseteq A \subseteq F^{k}$, and the corresponding lattices of flats, ordered by inclusion, are isomorphic.

(16) For any integer $l, 0 \leq l \leq r(M)$, let $g^{\prime}=\{J \subseteq E: J \in 9$ and $|J| \leq l\}$. Then $M^{\prime}=\left(E, g^{\prime}\right)$ is a matroid having rank function $r^{\prime}(A)=\min (l, r(A))$, called the $l$ truncation of $M$, and denoted by $M^{(l)}$. The flats of $M^{(l)}$ are just the flats of $M$ having rank at most $l-1$, and $E$.

A matroid may also be characterized by its circuit-family in the following manner.

(17) A family $\mathcal{C}$ of subsets of a finite set $E$ is the circuit-family of a matroid on $E$ if and only if

(C1) $\varnothing \notin \mathcal{C}$ and no member of $\mathcal{C}$ is a proper subset of another.

(C2) For any two distinct members $C_{1}$ and $C_{2}$ of $\mathcal{C}$ and $x \in C_{1} \cap C_{2}$ there exists $C \in \mathcal{C}$ such that $C \subseteq C_{1} \cup C_{2}-\{x\}$.

(18) Given a matroid $M$ on the set $E$ with basis-family $\Re$, the dual of $M$, denoted by $M^{*}$, is the matroid with basis-family $\mathfrak{B}^{*}=\{E-B: B \in \mathfrak{B}\}$.

(19) By (5), the hyperplanes of $M$ are the maximal subsets of $E$ containing no basis of $M$. Hence the set-complements of the hyperplanes of $M$ are the circuits of the dual, $M^{*}$. We shall also refer to them as the cocircuits of $M$.

(20) If $M$ is a matroid design, then the cocircuits all have the same cardinality, $c^{*}(M)=|E|-k(M)$, called the cocircuit cardinality of $M$.

Using (17), we may characterize the hyperplane-family of a matroid as follows.

(21) A family $\mathcal{H}$ of subsets of a finite set $E$ is the hyperplane-family of a matroid on the set $E$ if and only if

(H1) $E \notin \mathcal{H}$ and no member of $\mathcal{H}$ is a proper subset of another.

(H2) For any two distinct members $H_{1}$ and $H_{2}$ of $\mathcal{H}$, and any $x \in E,\{x\} \cup$ $\left(H_{1} \cap H_{2}\right)$ is contained in some member of $\mathcal{H}$.

(22) Actually, the following stronger version of (H2) holds for the hyperplanefamily of a matroid $M=(E, \mathcal{H})$.

$(\mathrm{H} 2)^{\prime}$ For any distinct $H_{1}, H_{2} \in \mathcal{H}$ and any $x \in E-\left(H_{1} \cup H_{2}\right), y \in H_{1}-H_{2}$, there exists $H \in \mathcal{H}$ such that $y \notin H$ and $\{x\} \cup\left(H_{1} \cap H_{2}\right) \subseteq H$.

A separator of a matroid $M=(E, 9)$ is a subset $S$ of $E$ such that for any circuit $C$ of $M, C \subseteq S$ or $C \subseteq E-S$. 
(23) It is evident from the definition that the intersection of any two separators is a separator, and the complement of a separator is a separator. Therefore, the minimal nonempty separators of $M$ partition $E$. Where $S$ is such a separator, $M \cdot S$ is called a component of $M . M$ is called connected if $M$ is its sole component.

The following theorem is due to Whitney [12].

(24) Theorem. $M=(E, g)$ is connected if and only if every two elements of $E$ are contained in a common circuit.

In view of (24) we say that a matroid is totally disconnected if it contains no circuits, i.e., if its independent sets form a Boolean algebra.

It may be shown [12] that the separators of a matroid $M$ and its dual $M^{*}$ are identical. Hence $M$ is connected if and only if $M^{*}$ is, and (22), (23) and (24) are valid with "circuit" replaced by "cocircuit". In the sequel, we shall sometimes use (24), stated for cocircuits, as the most convenient criterion for verifying connectedness. Using the relation between cocircuits and hyperplanes, we also have the following criterion.

(25) $M=(E, \mathcal{H})$ is connected if and only if for every $x, y \in E$ there exists $H \in \mathcal{H}$ such that $x \notin H$ and $y \notin H$.

(26) We note that any matroid $M$ is determined by its components, for if $M_{i}=$ $\left(s_{i}, \mathcal{H}_{i}\right), i=1, \cdots, t$, are the components of $M$, then, by (12), the hyperplanes of $M$ are precisely the sets of form $H \cup \bigcup_{i=1 ; i \neq k}^{t} S_{i}$, where $H \in \mathcal{H}_{k}$.

From (13) we may also conclude the following.

(27) Every component of a matroid design is a matroid design.

We have seen that a matroid may be characterized by its independent sets, its circuits, or its hyperplanes. In the following sections, however, we shall usually find it most convenient to present matroids "geometrically" in terms of their hyperplanes, or more generally, their flats. Correspondingly we shall use the properties of cocircuits to deal with questions of connectedness.

Let $M=(E, \mathcal{H})$ be any matroid.

(28) The 0-flat of $M, F^{0}$, is unique and consists of all elements not contained in any basis of $M$. Such elements are called loops. By the definition of closure, $F^{0}$ is a subset of every flat. Hence we may exclude all loops from $M$ without essentially altering the set-relationships among the various flats. To simplify the presentation of later results, we shall therefore always assume in the sequel that $M$ has no loops.

(29) The 1-flats, or points, of $M$ are analogous to points of a projective geometry in the sense that every flat $F$ is partitioned by the points that meet it. This follows immediately from (4) and the assumption that the 0 -flat of $M$ is null. An $m$-point is a point having cardinality $m$, and it is said to be a simple point if $m=1$. In general, a simple flat is a flat whose cardinality equals its rank. A simple matroid is a matroid all of whose points are simple. 
(30) A 2-flat of $M$ is called a line. Any two distinct points are contained in a unique line.

The following theorem expresses a basic fact about the structure of flats in a matroid.

(31) Theorem. Let $M$ be a rank $n$ matroid. For any i-flat $F^{i}$ and $k$-flat $F^{k}$ of $M$ such that $F^{i} \varsubsetneqq F^{k}, 0 \leq i<k \leq n, F^{k}-F^{i}$ is partitioned by the sets of form $F^{i+1}-F^{i}$, where $F^{i+1}$ is an $(i+1)$-flat containing $F^{i}$ and contained in $F^{k}$.

Examples of matroid designs: perfect matroid designs. We shall now present three general classes of matroid designs that have the following additional regularity property: for any positive integer $j$, the flats of rank $j$ all have the same cardinality, $\alpha(j)$. Such matroids are a special case of matroid designs and are called perfect matroid designs (PMD's). PMD's enjoy a high degree of regularity, as the following result shows. (For proofs of the next few theorems and a detailed treatment of PMD's, the reader is referred to [15].)

(32) Theorem. Let M be a PMD. For any i-flat $F^{i}$ and $k$-flat $F^{k}$ of $M$ such that $F^{i} \subseteq F^{k}$, the number $t_{M}(i, j, k)$ of j-flats $F^{j}$ sucb that $F^{i} \subseteq F^{j} \subseteq F^{k}$ depends only on $i, j$, and $k$.

Any PMD $M$ may be very conveniently described parametrically by the numbers $d_{i}=t_{M}(0,1, i)-t_{M}(0,1, i-1), 1 \leq i \leq r(M)$. The sequence $\left(d_{n}, d_{n-1}, \cdots, d_{1}\right)$ is called the $d$-sequence of $M$, and denoted by $d(M)$. The function $t_{M}(i, j, k)$, $0 \leq i \leq j \leq k \leq r(M)$, is determined from the $d$-sequence of $M$ by the relation

$$
t_{M}(i, j, k)=\left(\prod_{l=i+1}^{j} \sum_{m=l}^{k} d_{m}\right) /\left(\prod_{l=i+1}^{j} \sum_{m=l}^{j} d_{m}\right) .
$$

(34) Theorem. Necessary conditions for a sequence $\left(d_{n}, d_{n-1}, \cdots, d_{1}\right)$ to be the d-sequence of a PMD are

(D0) $d_{1}=1$ and $d_{i} \geq 1,1 \leq i \leq n$.

(D1) $\left(\Pi_{l=i+1}^{j} \Sigma_{m=l}^{k} d_{m}\right) /\left(\Pi_{l=i+1}^{j} \Sigma_{m=l}^{j} d_{m}\right)$ is an integer, $0 \leq i \leq j \leq k \leq n$.

(D2) $d_{i-1} \mid d_{i}, 2 \leq i \leq n$, and $d_{i+1} / d_{i} \geq d_{i} / d_{i-1}, 2 \leq i . \leq n-1$.

(D2) is derived from Fisher's inequality for block designs.

We now turn to some examples of PMD's. We shall also observe how various classes of PMD's are determined by the form of their $d$-sequences.

Let $M=(E, \mathcal{H})$ be a matroid, and a a positive integer. For each element $x \in E$ choose a set $S_{x}$ of a elements in such a way that for distinct elements $x, x^{\prime} \in E, S_{x} \cap S_{x^{\prime}}=\varnothing$. Let $E^{\prime}=\bigcup_{x \in E} S_{x}$, and let $\mathcal{H}^{\prime}=\left\{\bigcup_{x \in H} S_{x}: H \in \mathcal{H}\right\}$. Clearly, $M^{\prime}=\left(E^{\prime}, \mathcal{H}^{\prime}\right)$ satisfies (H1) and (H2), hence $M^{\prime}$ is a matroid. $M^{\prime}$ is said to be an a-inflation of $M$, and $M$ is an a-deflation of $M^{\prime}$. Now $F^{\prime}$ is a flat of $M^{\prime}$ if and only if $F^{\prime}=\bigcup_{x} \in F S_{x}$ for some flat $F$ of $M$. Thus the lattices of a 
matroid and its inflation (or deflation) are isomorphic.

For any integers $v$ and $k$ such that $v>k \geq 0$, there exists a matroid design $M$ on $v$ elements with hyperplane size $k$ that is, in addition, a perfect matroid design. Namely, let $M_{E}$ denote the totally disconnected matroid on a $v$-set $E$, and let $M=M_{E}^{(k+1)}$ be the $(k+1)$-truncation of $M_{E}$. Where $a$ is a positive integer, any $\alpha$-inflation $M(\alpha)$ of such an $M$ is called an $(\alpha, k, v)$-trivioid, denoted by $\sigma(\alpha, k, v)$, and $M(\alpha)$ is said to be a trivial matroid design. The hyperplanes of $M(\alpha)$ are precisely the subsets of $E$ that are unions of any $k$ distinct points.

(35) Theorem. Every sequence of form $\left(d_{n}, 1,1, \ldots, 1\right)$ baving length $n$ is the d-sequence of an $\left(\alpha, n-1, d_{n}+n-1\right)$-trivioid, and conversely.

In later sections our aim will be to show that for certain integers $\gamma$, every matroid design with cocircuit cardinality $\gamma$ is trivial.

A second class of matroid designs arises from the so-called $t$-designs, or Steiner systems.

A $t$-design, or more specifically, a $t$ - $(v, k, \lambda)$ design, $(V, W)$, is a $v$-set $V$ and a system 70 of $k$-subsets of $V, k<v$, called blocks, such that every $t$-subset of $V, t \geq 2$, is contained in exactly $\lambda$-blocks. (Repeated blocks are admissible in the system 20 .) We shall sometimes refer to any such pair $(V, \hat{i})$ for short as a $D_{t}(v, k, \lambda) . A$ BIBD is precisely a 2 -design.

The following result is proved in [15].

(36) Theorem. For any integer $t \geq 2$, the byperplanes of a matroid $M$ are the blocks of a $t-(v, k, 1)$ design with $k<v$ if and only if $M$ is a simple rank $t+1$ PMD with d-sequence $(v-k, k-t+1,1, \ldots, 1)$.

A third class of matroid designs consists of the classical finite projective and affine geometries. We shall denote a finite projective geometry and a finite affine geometry of dimension $n$ and order $s$ by $\operatorname{PG}(n, s)$ and $\operatorname{EG}(n, s)$, respectively. Then we have the following result.

(37) Theorem. For any integers $n \geq 2$, and $s \geq 2$ :

(i) every simple, rank $n+1 P M D$ with d-sequence $\left(s^{n}, s^{n-1}, \ldots, s, 1\right)$ is a PG $(n, s)$, and conversely;

(ii) every simple, rank $n+1$ PMD with d-sequence $\left(s^{n-1}(s-1)\right.$, $\left.s^{n-2}(s-1), \ldots, s-1,1\right)$ is an $\mathrm{EG}(n, s)$, and conversely.

This theorem may be derived from an extension of the Dembowski-Wagner theorem [4] due to W. Kantor [9]. For an alternate proof, see [15].

Further examples of matroid designs. Next we shall present several constructions of matroid designs that are not PMD's.

Let $M=(E, \mathcal{H})$ be any rank $n$ matroid (not necessarily a matroid design), and let $\mathcal{F}_{n-2}$ be the set of $(n-2)$-flats of $M$. For a given positive integer $m$, 
let $P(m)$ denote the rank 1 matroid consisting of a single point on $m$ elements (we shall identify $P(m)$ with the set of $m$ elements itself), and choose $P(m)$ disjoint from $E$. Then it may easily be verified that $\mathcal{H}^{\prime}=\mathcal{H} \cup\left\{P(m) \cup F: F \in \mathcal{F}_{n-2}\right\}$ satisfies (H1) and (H2); hence $\mathcal{H}^{\prime}$ is the family of hyperplanes of a matroid on the set $E^{\prime}=E \cup P(m)$. ( $\left(E^{\prime}, \mathcal{H}^{\prime}\right)$ is called the one-point extension of $M$ by $P(m)$, and denoted by $M \oplus P(m)$. Suppose now that $M$ is a matroid design, $k(M)=k$, and suppose further that the $(n-2)$-flats of $M$ all have cardinality $b<k$. Then clearly $M \oplus P(k-b)$ will be a matroid design with hyperplane size $k$. This construction, due to U.S. R. Murty, plays an important part in the classification of matroid designs, as we shall presently show.

$\mathrm{PG}(2,2) \oplus P(2)$ is shown in Figure 1 . In this and subsequent matroid diagrams, the simple points will be represented by solid nodes, and points with $m>1$ elements will be represented by a hollow node containing the number $m$. 2-flats will be represented by lines, and 3-flats by planes, where convenient.

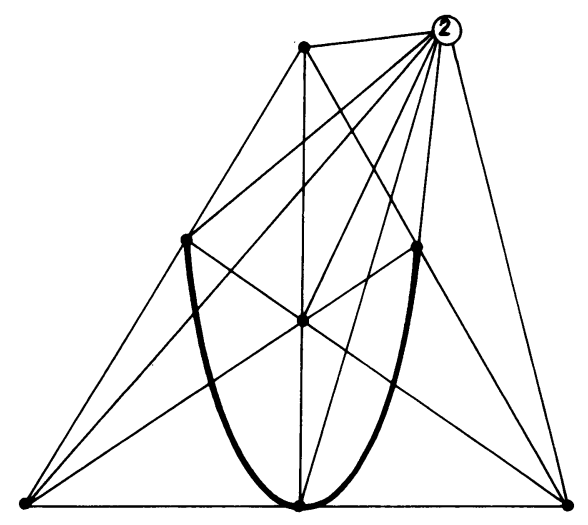

Figure 1. The matroid design $P G(2,2) \oplus P(2)$

An interesting problem is to construct a simple matroid design (i.e., one with singleton points) that is not a perfect matroid design. Such designs are surprisingly rare. We shall now describe such a design having the fewest possible number of points; in a subsequent section several infinite classes of them are described.

(38) Let $L_{1}, L_{2}$ be disjoint sets of three elements each, and let $Q$ be the set of all nine pairs $\{x, y\}$ such that $x \in L_{1}, y \in L_{2}$. Let $Q$ be partitioned into three sets $P_{1}, P_{2}, P_{3}$, each containing three disjoint pairs.

Now let $A$ be a set of four elements disjoint from $L_{1} \cup L_{2}$, and let $Q^{\prime}$ be the set of six distinct pairs $\{x, y\}$ in $A$. Let $P_{1}^{\prime}, P_{2}^{\prime}, P_{3}^{\prime}$ be a partitioning of $Q^{\prime}$ into three sets, each containing two disjoint pairs of $Q^{\prime}$. We define

$$
\begin{aligned}
E & =A \cup L_{1} \cup L_{2}, \\
Y_{i} & =\left\{L_{i} \cup\{y\}: y \in E-L_{i}\right\}, \quad \text { for } i=1,2, \\
Z_{j} & =\left\{q \cup q^{\prime}: q \in P_{j}, q^{\prime} \in P_{j}^{\prime}\right\}, \quad j=1,2,3,
\end{aligned}
$$


and

$$
\mathcal{H}=\left(\bigcup_{j=1}^{3} Z_{j}\right) \cup\left(\bigcup_{i=1}^{2} Y_{i}\right) \cup\{A\} .
$$

It is straightforward to verify that $(E, \mathcal{H})$ satisfies $(\mathrm{H} 1)$ and $(\mathrm{H} 2)$, hence it is a matroid. We shall denote any matroid of this form by $\Psi$. By construction, $\Psi$ is a matroid design, and $k(\Psi)=r(\Psi)=4$. The lines of $\Psi$ are precisely the sets of form $L=H_{1} \cap H_{2}$ such that $\left|H_{1} \cap H_{2}\right| \geq 2$ and $H_{1}, H_{2}$ are distinct members of $\mathcal{H}$. In particular, $L_{1}$ and $L_{2}$ are lines of $\Psi$, and every other line of $\Psi$ is simple. Therefore $\Psi$ is not a PMD. Finally, for every two distinct elements $x, y \in E$, there is a hyperplane containing $x$ but not $y$, so $\Psi$ is simple.

Representative lines and planes of $\Psi$ are shown in Figure 2.

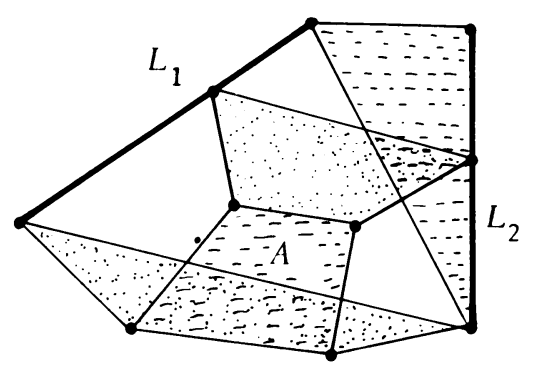

Figure 2. The matroid design $\Psi$

The structure of matroid designs. We have presented the above examples to suggest the variety among matroid designs. For more complex examples, the reader is referred to [15]. But our main object in this paper is to show that matroid designs are still such highly regular objects that it is often possible to completely describe the structure of all matroid designs with given rank and cocircuit cardinality. This problem in its generality we shall call the characterization problem for matroid designs.

(39) We shall first observe that to describe the possible structures of matroid designs, we will not sacrifice any substantial results by restricting our attention to connected matroid designs, for by (27) every component of a matroid design is also a matroid design and by (26) the components of a matroid determine the matroid completely. Thus we define $\prod_{n}(\gamma)$ for positive integers $n$ and $\gamma$ to be the family of all connected matroid designs $M$ such that $r(M)=n$ and $c^{*}(M)=\gamma$. We shall seek to characterize $\mathbb{M}_{n}(\gamma)$ for various values of $n$ and $\gamma$.

(40) Second, let us recall from (12) that, for any subset $A$ of $E$, the hyperplanes of $M \cdot(E-A)$ are precisely the sets of form $H-A$ where $H$ is a hyperplane of $M$ containing $A$. Therefore, the cocircuits of $M \cdot(E-A)$ are precisely the cocircuits of $M$ not meeting $A$. In particular, $c^{*}(M)=c^{*}(M \cdot(E-A))$.

(41) Further, let us note that, by (31), every hyperplane of $M$ is partitioned by 
the points that meet it; hence also every cocircuit of $M$ is partitioned by the points that meet it.

The next theorem establishes a fundamental property of connected matroid designs.

(42) Theorem. If $M$ is a connected matroid design and $F$ is a j-flat of $M$ $(0 \leq j \leq n)$, then $M \cdot(E-F)$ is a connected matroid design.

Proof. (13) says that $M \cdot(E-F)$ is a matroid design. The essence of the theorem is that connectivity is preserved under the contraction of a matroid design to the complement of a flat.

(43) Suppose that (42) has been proved whenever $r(F)<j, j \geq 2$, and let $F^{j}$ be a $j$-flat of $M$. Where $F^{j-1}$ is a $(j-1)$-flat contained in $F^{j}$, we have

$$
\begin{aligned}
M \cdot\left(E-F^{j}\right) & =\left(M \cdot\left(E-F^{j-1}\right)\right) \cdot\left(E-F^{j}\right) \\
& =\left(M \cdot\left(E-F^{j-1}\right)\right) \cdot\left(\left(E-F^{j-1}\right)-\left(F^{j}-F^{j-1}\right)\right) .
\end{aligned}
$$

Now $M \cdot\left(E-F^{j-1}\right)$ is connected, by the induction hypothesis (43), and $F^{j}-F^{j-1}$ is a point of $M \cdot\left(E-F^{j-1}\right)$. Hence $M \cdot\left(E-F^{j}\right)$ is connected, again by (43). Therefore, it suffices to prove (42) when $F$ is a point of $M$. (If $F$ is a 0 -flat there is nothing to prove.)

(44) Let $F$, then, be a point of $M$, and let $c^{*}(M)=\gamma$. We shall show that for every $x, y \in E-F, x \neq y$, there is a cocircuit $C$ such that $\{x, y\} \subseteq C$ and $C \cap F=\varnothing$, and it will follow from (24) and (40) that $M \cdot(E-F)$ is connected.

With $x, y$, and $F$ as above, $2 \leq r(F \cup\{x, y\}) \leq 3$, since $F$ is closed and $r(F)=1$. If $r(F \cup\{x, y\})=2$, then for any $w \in F,\{w, x\}$ is a basis of $F \cup\{x, y\}$. Where $B$ is any basis of $M$ containing $\{w, x\}, \operatorname{cl}(B-\{x\})=H$ is a hyperplane containing $F$, but $y \notin H$ because $x$ depends on $\{w, y\}$ and $x \notin H$. Therefore $E-H$ is a cocircuit as required by (44).

We may suppose then that $r(F \cup\{x, y\})=3$, i.e., that $\{w, x, y\}$ is independent for any $w \in F$. We shall show the existence of two cocircuits $C_{1}$ and $C_{2}$ such that $\{x, y\} \subseteq C_{1} \cup C_{2}-C_{1} \cap C_{2}$ and $F \subseteq C_{1} \cap C_{2}$. If $x, y$, and $F$ are contained in some common cocircuit, $C_{1}$, choose $w \in F$, and let $B$ be a basis of $M$ extending $\{w, x, y\}$. Then we take $H=\operatorname{cl}(B-\{w\})$ and $C_{2}=E-H$. If $x, y$, and $F$ are contained in no common cocircuit we let $C_{1}$ and $C_{2}$ be cocircuits containing $\{x\} \cup F$ and $\{y\} \cup F$ respectively (using (24), (41), and the hypothesis that $M$ is connected).

With $C_{1}$ and $C_{2}$ as above, there exist, by (C2), cocircuits $C_{x}$ and $C_{y}$ such that $x \in C_{x} \subseteq C_{1} \cup C_{2}-F$ and $y \in C_{y} \subseteq C_{1} \cup C_{2}-F$. Thus $C_{x} \cup C_{y} \subseteq C_{1} \cup C_{2}$ - $F$; since $\left|C_{x}\right|=\left|C_{y}\right|=\left|C_{1}\right|=\left|C_{2}\right|=\gamma$ and $F \neq \varnothing$, we must have $C_{x} \cap C_{y} \neq \varnothing$. But then $M^{\prime}=M \cdot\left(C_{x} \cup C_{y}\right)$ is connected, by definition. Thus, by (24), there is a cocircuit $C$ of $M^{\prime}$ containing $x$ and $y$, and, by (40), $C$ is a cocircuit of $M$ disjoint from $F . \square$ 
(45) Corollary. If $M \in M_{n}(\gamma)$ and $F$ is a j-flat of $M$, then $M \cdot(E-F) \in M_{n-j}(\gamma)$.

This result follows at once from (11), (40), and (42).

The contraction, $M \cdot(E-F)$, of a matroid $M$ to the complement in $E$ of a flat $F$ will be called the contraction of $M$ at $F$. We shall sometimes abbreviate $M \cdot(E-F)$ to $M_{F}$. in this case. In general, it is not true that the contraction at a flat of a connected matroid is again connected.

The trivioids of a given rank and cocircuit cardinality are particularly easy to characterize, as the following theorem shows (the proof is left to the reader).

(46) Theorem. For any positive integers $n$ and $\gamma$, the trivioids in $\prod_{n}(\gamma)$ are precisely the trivioids of form $\sigma(\alpha, n-1, \gamma / \alpha+n-1)$, where $\alpha \mid \gamma$ and $\alpha<\gamma$.

Our next two theorems establish conditions under which a matroid design is a trivioid.

(47) For any matroid $M=(E, \mathcal{H})$ we let $P(M)$ denote the set points of $M$. Where $A \subseteq E, P(A)$ shall denote the set of points of $M$ contained in $A$.

(48) Theorem. Let $M=(E, \mathcal{H})$ be a connected matroid design. If for some point $b$ of $M, M \cdot(E-b)$ is $a(|b|,-,-)$-trivioid and $P(M \cdot(E-b)) \subseteq P(M)$, then $M$ is a $(|b|,-,-)$-trivioid.

Proof. Let $M \cdot(E-b)$ be a $\left(|b|, k^{\prime}, v^{\prime}\right)$-trivioid. We shall show that for every set $W$ of $k^{\prime}+1$ distinct points of $M, \bigcup_{X \in W} X \in \mathcal{H}$.

(49) If $b \in W$, then $W-\{b\}$ is a set of $k^{\prime}$ points of $M \cdot(E-b)$, so $\bigcup_{X \in W-\{b\}} X$ is a hyperplane of $M \cdot(E-b)$, and thus $\bigcup_{X \in W} X$ is a hyperplane of $M$.

(50) If $b \notin W$, let $H$ be a hyperplane of $M$ not containing $b$ such that $|P(H) \cap W|$ is a maximum. There is such a hyperplane, for otherwise $b$ would be a separator of $M$ (unless $r(M)=1$, in which case $E=b$ and $M$ is a $(|b|, 0,1)$-trivioid).

(51) Suppose that $c \in W-P(H)$ for some point $c$. Since $|P(H)|=|W|=k^{\prime}+1$, there is a point $d \in P(H)-W$, and thus $W^{\prime}=P(H) \cup\{b\}-\{d\}$ has cardinality $k^{\prime}+1$. Then $H^{\prime}=\bigcup_{X \in W^{\prime}} X$ is a hyperplane of $M$, by (49). From (H2) of (22) we conclude that there is a hyperplane $H^{\prime \prime}$ of $M$ such that $H^{\prime \prime} \supseteq c \cup\left(H^{\prime} \cap H\right)$ and $b \cap H^{\prime \prime}=\varnothing$. But then $\left|W \cap P\left(H^{\prime \prime}\right)\right|>|W \cap P(H)|$, contradicting the choice of $H$. Therefore $W-P(H)=\varnothing$; that is, $H=\bigcup_{X \in W} X$.

(52) In (29) we defined a simple $j$-flat of a matroid to be a $j$-flat having cardinality $j$. We say that a $j$-flat is elementary if it contains precisely $j$ points of the matroid.

(53) Theorem. Let $M=(E, H)$ be a connected, nontrivial matroid design. If there exist two distinct points $a_{1}$ and $a_{2}$ of $M$ such that $M \cdot\left(E-a_{1}\right)$ and $M \cdot\left(E-a_{2}\right)$ are trivial, then $r(M)=3$. Conversely, if $r(M)=3$ then $M \cdot(E-a)$ is trivial for every point $a$ of $M$. 
Proof. Suppose that $r(M)=3$. For any point $a$ of $M, r(M \cdot(E-a))=2$, so the hyperplanes of $M \cdot(E-a)$ are its points, and each has cardinality $a=k(M)-|a|$. Thus $M \cdot(E-a)$ is an $(\alpha, 1,|E-a| / \alpha)$-trivioid.

Conversely, suppose that $M$ is a nontrivial connected matroid design such that $k(M)=\dot{k}$ and $M_{i}=M \cdot\left(E-a_{i}\right)$ is a trivioid for distinct points $a_{i}$ of $M$, $i=1,2$. Let the points of $M_{i}$ have cardinality $s_{i} \cdot r(M)=n \geq 3$, since every matroid design of rank less than 3 is trivial.

(54) Let $\mathcal{Q}_{i}$ be the set of lines of $M$ containing $a_{i}$, for $i=1,2$, and let $L_{12}$ be the unique line containing $a_{1} \cup a_{2}$. By (12) the points of $M_{i}$ are precisely the sets of form $L-a_{i}$, where $L \in \mathfrak{I}_{i}$. Hence $\left|L-a_{i}\right|=s_{i}$ for every $L \in \mathfrak{I}_{i}$, and the union of every $n-2$ distinct sets of the form $L-a_{i}$ is a hyperplane of $M_{i}$. Therefore, by $(12)$, the union of every $(n-2)$ distinct members of $\mathcal{L}_{i}$ is a hyperplane of $M$, so

$$
(n-2) s_{1}+\left|a_{1}\right|=(n-2) s_{2}+\left|a_{2}\right|=k .
$$

(56) Further, since the hyperplanes are proper subsets of $E$, each $\mathscr{L}_{i}$ contains at least $(n-1)$ lines, and the members of $\mathcal{L}_{i}$ are themselves proper subsets of $E$.

(57) Let us now assume, contrary to the theorem, that $r(M)=n \geq 4$. Suppose in addition that for some line $L_{0} \in \mathcal{P}_{2}$ different from $L_{12}, L_{0}$ contains three distinct points $a_{2}, b$, and $c$. Clearly, $a_{1} \not L_{0}$, so every point $a$ of $L_{0}$ is the intersection of $L_{0}$ with a unique line $L_{a} \in \mathscr{I}_{1}$. Let $L_{b}$ and $L_{c}$ be the lines of $\mathcal{L}_{1}$ corresponding to $b$ and $c$ in this way. Then, by (56), there exists an $(n-2)$-subset $\mathscr{Q}^{\prime}$ of $\mathscr{Q}_{1}$ containing $L_{b}$ and $L_{c}$ and omitting $L_{12}$. Hence $H=\bigcup_{L \in \mathfrak{l}} L \in \mathcal{H}$ and $H$ contains $b \cup c$, but $H \cap a_{2}=\varnothing$, so $H$ does not contain $L_{0}=\operatorname{cl}(b \cup c)$, which is absurd. Hence, if $n \geq 4$, every line in $\varrho_{2}-\left\{L_{12}\right\}$ is elementary. Likewise, every line in $\bigodot_{1}-\left\{L_{12}\right\}$ is elementary.

(58) Where $b$ is any point of $M$ not contained in $L_{12}$, it follows from the

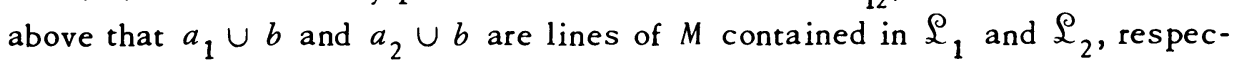
tively. Hence $b$ is a point of $M_{1}$ and of $M_{2}$. Therefore $s_{1}=s_{2}=|b|$, so (55) implies that $\left|a_{1}\right|=\left|a_{2}\right|$. Let $s_{1}=s_{2}=\sigma,\left|a_{1}\right|=\left|a_{2}\right|=\alpha$.

Since every hyperplane of $M$ contains at least $n-1$ points, and since, by (25) and the assumption that $M$ is connected, there exists a hyperplane of $M$ disjoint from $a_{1} \cup a_{2}$, it follows from (58) that $(n-1) \sigma \leq k$. By (55), $(n-2) \sigma+\alpha=k$, and so $\sigma \leq \alpha$. On the other hand, $a_{2} \subseteq L_{12}-a_{1}$, and so $a=\left|a_{2}\right| \leq\left|L_{12}-a_{1}\right|=\sigma$. Therefore $a=\sigma$, and $L_{12}=a_{1} \cup a_{2}$. Hence $M \cdot\left(E-a_{1}\right)$ is an $\left(\left|a_{1}\right|,-,-\right)$ trivioid whose points are points of $M$, so $M$ is an $\left(\left|a_{1}\right|,-,-\right)$-trivioid, by (48), contrary to our hypothesis on $M$. Therefore $r(M)=3$.

(59) Theorem. If, for some $n \geq 3$ and $\gamma \geq 1$, there exists a nontrivial matroid $M \in M_{n}(\gamma)$, then for every $m, 3 \leq m \leq n$, there exists a nontrivial matroid $M_{m} \in \mathbb{M}_{m}(\gamma)$. 
Proof. Let $m$ be the greatest integer such that $m \leq n$ and $\mathbb{M}_{m}(\gamma)$ contains a nontrivial member $M_{m}=\left(E_{m}, \mathcal{H}_{m}\right)$ and every member of $\mathbb{M}_{m-1}(\gamma)$ is trivial. By (45), $M_{m} \cdot\left(E_{m}-a\right) \in M_{m-1}(\gamma)$ for every point $a$ of $M_{m}$; hence by $(53), r\left(M_{m}\right)=3$, that is, $m=3$.

Every connected rank 1 matroid design is a trivioid, since $\varnothing$ is the only hyperplane. Likewise every connected rank 2 matroid design is a trivioid, for the hyperplanes are identical with the points. Therefore, for every $\gamma, \mathbb{R}_{1}(\gamma) \cup \mathbb{M}_{2}(\gamma)$ contains only trivial matroids.

(60) It follows then from (59) that every connected matroid design with cocircuit cardinality $\gamma$ is trivial if and only if every rank 3 connected matroid design with cocircuit cardinality $\gamma$ is trivial. More generally, we shall find that if there exist only a few nonisomorphic matroids in $\mathbb{M}_{3}(\gamma)$, then it is frequently possible to describe completely the members of $\mathbb{M}_{n}(\gamma)$ for every $n$. Hence we shall turn to the study of rank 3 matroid designs in detail.

For a given cocircuit cardinality $\gamma$, suppose there exists $M=(E, \mathcal{H}) \in \mathbb{M}_{3}(\gamma)$, and let $k(M)=k$.

(61) For any point $a$ of $M$, let $\mathcal{H}_{a}$ denote the set of lines (i.e., hyperplanes of $M)$ containing $a$. By (31), the sets $H-a$ such that $H \in H_{a}$ partition $E-a$, and hence $(k-|a|) \mid(|E|-|a|)$, or, noting that $|E|-k=\gamma,(k-|a|) \mid \gamma$.

(62) Suppose that $k-|a|=\gamma$ for some point $a$. Then $(|E|-|a|) /(k-|a|)=2$, so $a$ is contained in precisely two lines of $M$, say $H_{1}$ and $H_{2}$. Let $P\left(H_{1}\right)=$ $\left\{a, a_{1}, \ldots, a_{u}\right\}$ and $P\left(H_{2}\right)=\left\{a, b_{1}, \cdots, b_{u}\right\} . H_{1}$ and $H_{2}$ are lines whose union is $E$, and since every two points are contained in a unique line, $a_{i} \cup b_{j}$ is a line for every $i$ and $j$. Thus

$$
k=|a|+\sum_{i=1}^{u}\left|a_{i}\right|=|a|+\sum_{j=1}^{v}\left|b_{j}\right|=\left|a_{i}\right|+\left|b_{j}\right|
$$

for $1 \leq i \leq u, 1 \leq j \leq v$.

From (63) it follows readily that $\left|a_{1}\right|=\left|a_{2}\right|=\cdots=\left|a_{u}\right|$ and $\left|b_{1}\right|=\left|b_{2}\right|=\cdots$ $=\left|b_{v}\right|$. Let $a$ be the cardinality of the $a_{i}$ 's and $\beta$ the cardinality of the $b_{i}$ 's. Then

$$
k=|a|+u \alpha=|a| \dot{+} \nu \beta=\alpha+\beta \text {. }
$$

Thus

$$
\beta=(u-1) \alpha+|a|, \quad|a|+u \alpha=|a|+v(u-1) \alpha+v|a|,
$$

and

$$
u a>v(u-1) a,
$$

which implies $v=1$. 
A symmetric analysis shows that $u=1$. Therefore $M$ contains only the three points $a, a_{1}$, and $b_{1}$, and $a$ is therefore a separator of $M$, contrary to the hypothesis that $M \in M_{3}(\gamma)$, i.e., that $M$ is connected. Hence

(64) $(k-|a|) \mid \gamma$ and $k-|a| \neq \gamma$ for every point $a$ of $M$.

For every $H \in \mathcal{H}$ and $d$, where $1 \leq d \leq k$, let $x_{d}^{H}$ denote the number of $(k-d)$ points contained in $H$, and let $\left\langle x^{H}\right\rangle$ denote the $k$-tuple of these numbers. From (64) it follows that

$$
|H|=k=\sum_{d \mid \gamma ; d \neq \gamma}(k-d) x_{d}^{H} .
$$

Where $a_{1}$ and $a_{2}$ are distinct points of $M$, (64) implies that $k-\left|a_{1}\right|=d_{1}$ and $k-\left|a_{2}\right|=d_{2}$ are proper divisors of $\gamma$. Since $\operatorname{cl}\left(a_{1} \cup a_{2}\right) \in \mathcal{H},\left|a_{1}\right|+\left|a_{2}\right| \leq k$; hence $\left|a_{2}\right| \leq d_{1}$ and

$$
k=\left|a_{2}\right|+d_{2} \leq d_{1}+d_{2} \leq 2 d,
$$

where $d$ is the largest proper divisor of $\gamma$. Thus we have proved the following

(67) Theorem. If $M \in)_{3}(\gamma), k(M)=k$, then

(i) For every byperplane $H$ of $M$ there is a solution $\left\langle x^{H}\right\rangle$ to the diophantine equation $k=\Sigma_{d \mid \gamma ; d \neq \gamma}(k-d) x_{d}$, such that $x_{d}^{H}$ is the number of $(k-d)$-points contained in $H$.

(ii) $k \leq 2 d$ where $d$ is the largest divisor of $\gamma$ less than $\gamma$.

We now have the necessary tools to determine all possible matroid designs with certain given ranks and cocircuit cardinalities.

Matroid designs with cocircuit cardinality a prime or prime squared.

(68) Theorem. If $p$ is a prime and $n \geq 0$, every member of $\pi_{n}(p)$ is trivial.

Proof. By (60), it suffices to prove (68) when $n=3$. Let $M \in \mathbb{M}_{3}(p), k(M)=k$. By (67)(ii), $k \leq 2$; hence $k=2$ (since every line contains two points). Therefore $M$ is a $(1,2,-)$-trivioid.

It is tempting to speculate that for $n \geq 3, M_{n}(\gamma)$ contains only trivioids if and only if $\gamma$ is a prime. However, this conjecture is false, as we shall subsequently show.

Another class of matroid designs that admits of relatively easy characterization is $\mathbb{M}_{3}\left(p^{2}\right)$, where $p$ is a prime.

(69) Theorem. If $p$ is a prime, every member of $\mathbb{M}_{3}\left(p^{2}\right)$ is either (i) a $\sigma\left(1,2, p^{2}+2\right)$; or (ii) a $\sigma(p, 2, p+2)$; or (iii) a PG $(2, p)$.

Proof. Let $M=(E, \mathcal{H}) \in \mathbb{M}_{3}\left(p^{2}\right)$, and let $k(M)=k$. The only proper divisors of $p^{2}$ are 1 and $p$. Hence, by (67), to every line $H$ of $M$ there corresponds a 
solution $\left(x^{H}, y^{H}\right)$ to the diophantine equation

$$
k=(k-1) x+(k-p) y
$$

such that $x^{H}$ and $y^{H}$ are the number of $(k-1)$-points and the number of $(k-p)$ points in $H$, respectively.

(71) Suppose that for some $H, x^{H}>0$. If $x^{H}=1$, then (70) says that $k=(k-1)$ $+(k-p) y^{H}$. Hence we must have $k=p+1, y^{H}=1$, and $|E|=\gamma+k=p^{2}+p+1$. With $k=p+1$, the only solutions to $(70)$ are $(x, y)=(1,1)$ and $(x, y)=(0, p+1)$; hence every line consists of either one $p$-point and one simple point, or $p+1$ simple points. Since every two points are contained in some line, $M$ contains at most one $p$-point, but, since $x^{H}=1, M$ contains exactly one $p$-point. Call it a. $E^{\prime}=E-a$ is then a union of $p^{2}+1$ simple points. Since every pair of simple points is contained in a unique line $L, L$ must contain only simple points, and hence $L \subseteq E^{\prime}$. Where $\mathcal{H}^{\prime}=\left\{L \in \mathcal{H}: L \subseteq E^{\prime}\right\}$ it follows that $\left(E^{\prime}, \mathcal{H}^{\prime}\right)$ is a $2-\left(p^{2}+1, p+1,1\right)$ design. But then $\left|\mathcal{H}^{\prime}\right|=\left(p^{2}+1\right) p^{2} /(p+1) p$, which is not an integer (unless $p=1$, in which case $(68)$ proves the result).

(72) Therefore, if $x^{H}>0$ for some $H$, then $x^{H} \geq 2$. Then, by (70), $k \geq(k-1) x^{H} \geq 2(k-1)$, which is only possible if $x^{\bar{H}}=2$ and $k=2$. In this case, $(x, y)=(2,0)$ is the only meaningful solution to (70), and hence every line of $M$ is the union of two simple points. Since $|E|=\gamma+k=p^{2}+2$, it follows that $M$ is a $\sigma\left(1,2, p^{2}+2\right)$, which is (i).

(73) Let us therefore assume that $x^{H}=0$ for every $H$. Then from (67) we have $k=(k-p) y^{H},(k-p)+p=(k-p) y^{H}$ and

$$
p=(k-p)\left(y^{H}-1\right) \text {. }
$$

Since $p$ is a prime, the only solutions to (74) are (I) $y^{H}=2, k=2 p$; and (II) $y^{H}=p+1, k=p+1$. In case (I) every line $H$ is the union of two $p$-points, and $|E|=2 p+\gamma=2 p+p^{2}=p(p+2)$; hence $M$ is a $\sigma(p, 2, p+2)$, which is (ii).

(75) In case (II), every line $H$ is the union of $(p+1)$ simple points and $|E|=p^{2}+p+1$. Thus $M$ is a simple $\operatorname{PMD}$ with $d$-sequence $\left(p^{2}, p, 1\right)$, so, by (37) $M$ is a $P G(2, p)$, which is case (iii).

Affine extension. Our next main object will be to determine $\mathbb{M}_{3}(p q)$ completely for certain pairs of distinct primes $p$ and $q$. To do so, however, we need to introduce a new class of matroids.

A matroid $M=(E, H)$ is said to be affine resolvable if there exists a partition of $\mathcal{H}$ into classes $\left\{\mathcal{P}_{i}, 1 \leq i \leq t\right\}$ such that each class $\mathscr{P}_{i}$ partitions $E$. The sets $\mathscr{P}_{i}$ are called parallel classes of $M$.

(76) Let $M$ be a rank 3 affine resolvable matroid with parallel classes $\mathcal{P}_{i}$, $1 \leq i \leq t, P(M)$ the set of points of $M$, and let $M^{\prime}=\left(E^{\prime}, \mathcal{H}^{\prime}\right)$ be a rank 2 or rank 3 
matroid with point set $P\left(M^{\prime}\right)=\left\{a_{1}^{\prime}, a_{2}^{\prime}, \ldots, a_{t}^{\prime}, a_{t+1}^{\prime}, \ldots, a_{u}^{\prime}\right\}$ such that $u \geq t$ and $E \cap E^{\prime}=\varnothing$. We define

$$
\begin{aligned}
X_{i} & =\left\{a_{i}^{\prime} \cup H: H \in \mathcal{P}_{i}\right\}, \quad 1 \leq i \leq t, \\
X & =\bigcup_{i=1}^{t} X_{i}, \\
Y & =\left\{a_{i}^{\prime} \cup a: a \in P(M), t+1 \leq i \leq u\right\},
\end{aligned}
$$

and let

$$
\mathcal{H}^{\prime \prime}=X \cup Y \cup \mathcal{H}^{\prime} \quad \text { if } r\left(M^{\prime}\right)=3, \quad \mathcal{H}^{\prime \prime}=X \cup Y \cup\left\{E^{\prime}\right\} \quad \text { if } r\left(M^{\prime}\right)=2 .
$$

In either case $\left(E \cup E^{\prime}, \mathcal{H}^{\prime \prime}\right)$ is called the affine extension of $M$ by $M^{\prime}$, and will be denoted by $M \ominus M^{\prime}$. We claim that $M \ominus M^{\prime}$ is a rank 3 matroid with point set $P^{\prime \prime}=P(M) \cup P\left(M^{\prime}\right)$. To establish this it suffices to show that every two members of $P^{\prime \prime}$ are contained in a unique member of $\mathcal{H}^{\prime \prime}$, a fact that may be readily verified. In addition, we claim that $M \ominus M^{\prime}$ is connected.

First, since $r(M)=3, M$ contains three distinct points, $\mathrm{a}, \mathrm{b}, \mathrm{c}$. The hyperplanes $\mathrm{cl}(a \cup b), \mathrm{cl}(b \cup c)$, and $\mathrm{cl}(a \cup c)$ must be contained in distinct parallel classes, hence $t \geq 3$. Now to establish connectedness, it suffices to show (by (25)) that for any two members, $a_{1}$ and $a_{2}$, of $P^{\prime \prime}$, there exists $H \in \mathcal{H}$ such that $a_{1} \cap H=a_{2} \cap H=\varnothing$. If $a_{1}, a_{2} \in P(M)$ then any member of $\mathcal{H}^{\prime}$ (or $E^{\prime}$ itself if $\left.r\left(M^{\prime}\right)=2\right)$ has this property. If $a_{1} \in P(M)$ and $a_{2} \in P\left(M^{\prime}\right)$, let $a_{2}^{\prime} \in P\left(M^{\prime}\right)-\left\{a_{2}\right\}$ and let $H_{a_{1} a_{2}^{\prime}}$ be the unique member of $\mathcal{H}^{\prime \prime}$ containing $a_{1}$ and $a_{2}^{\prime}$. Since

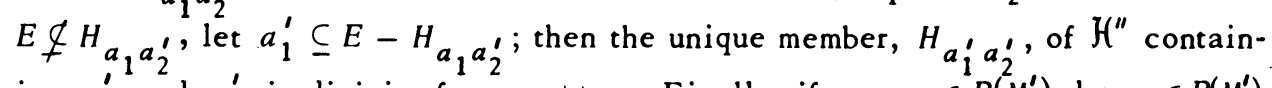
ing $a_{1}^{\prime}$ and $a_{2}^{\prime}$ is disjoint from $a_{1} \cup a_{2}$. Finally, if $a_{1}, a_{2} \in P\left(M^{\prime}\right)$, let $a_{3} \in P\left(M^{\prime}\right)$ $-\left\{a_{1}, a_{2}\right\}$; then any hyperplane containing $a_{3}$ and a point of $M$ has the desired property.

If, in (76), the cardinalities of the points and lines of $M$ and $M^{\prime}$ are appropriately related, then $M \ominus M^{\prime}$ will be a matroid design.

The construction (76) generalized the way in which a $\operatorname{PG}(2, s)$ is derived from an $\operatorname{EG}(2, s)$. Indeed, a $\operatorname{PG}(2, s)$ is just an $\operatorname{EG}(2, s) \ominus \sigma(1,1, s+1)$, as may easily be verified. However, there are many more possibilities. In Figure 3 we show two easy examples of affine extension that are not projective geometries.

(77) A very general class of affine extensions arises from certain complete subgraphs. In our terminology, a complete subgraph of order $n$ is just a $\sigma(1,2, n)$; in graph terminology the points are called vertices and the hyperplanes are called edges. It is a well-known result of graph theory that for every positive integer $m$, $\sigma(1,2,2 m)$ is affine resolvable into $2 m-1$ parallel classes. For any such $m$, $\sigma(1,2,2 m)$ and $\sigma(2,2,2 m-1)$ are both rank 3 matroids, and the latter has $2 m-1$ points, each of cardinality 2 . It follows that $\sigma(1,2,2 m) \ominus \sigma(2,2,2 m-1)$ is a connected rank 3 matroid, called the complete graph extension of order $m$, 
$\operatorname{CGE}(m)$. Figure 4 shows $\sigma(1,2,6), \sigma(2,2,5)$, and $\sigma(1,2,6) \ominus \sigma(2,2,5)$.

Rank 3 matroid designs with cocircuit cardinality $p q$, where $p$ and $q$ are distinct primes.

(78) Theorem. Let $p$ and $q$ be distinct primes such that either

(1) $p<2 q / 3+1$; or

(2) $2 q / 3+1 \leq p \leq 3 q / 4+1$

and the polynomial

$$
\Theta_{p, q}(x)=(q+1) x^{2}-\left(12 p q-2 q^{2}-6 p^{2}+6 p+3 q+5\right) x+6(p+1)(p q+q+1)
$$

bas no positive integral root.

Then if there exists a nontrivial matroid $M \in \mathbb{M}_{3}(p q)$ exactly one of the following bolds.

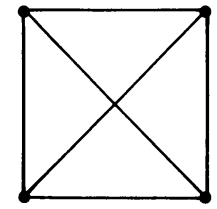

$\mathrm{EG}(2,2)$
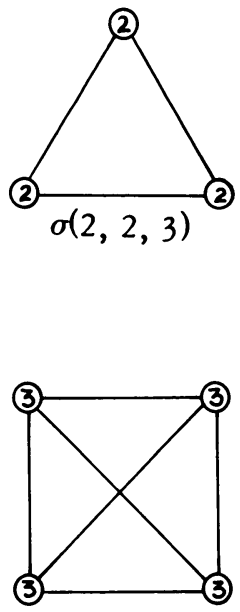

$M$ : a 3-inflation of EG $(2,2)$

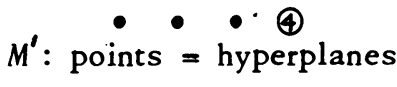

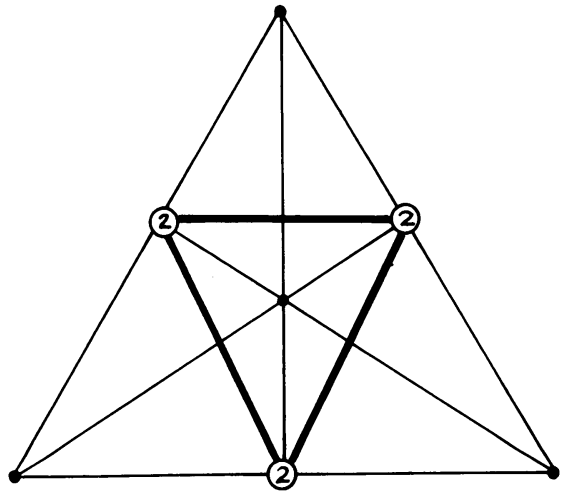

$\mathrm{EG}(2,2) \ominus \sigma(2,2,3)$

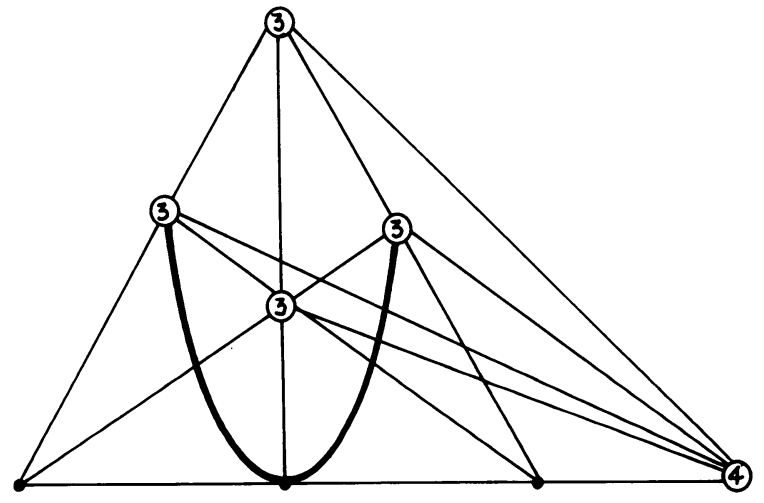

$M \ominus M^{\prime}$

Figure 3. Two examples of affine extension 
(i) $(p+1) \mid q(q+1)$ and $M$ is a $D_{2}(p q+p+1, p+1,1)$, i.e., $a 2-(p q+p+1$, $p+1,1)$ design.

(ii) $(p+1) \mid q(q-1)$ and $M$ is a $D_{2}(p q+1, p+1,1) \oplus P(p)$.

(iii) $q=2 p-1$ and $M$ is an $\operatorname{EG}(2, p) \ominus \sigma(p, 2, p+1)$.

(79) Proof. Let $p, q$, and $M=(E, \mathcal{H})$ satisfy the hypothesis of (78). Since $r(M)=3, \mathcal{H}$ is the family of lines of $M$. By $(53), M_{a}=M \cdot(E-a)$ is a trivioid for
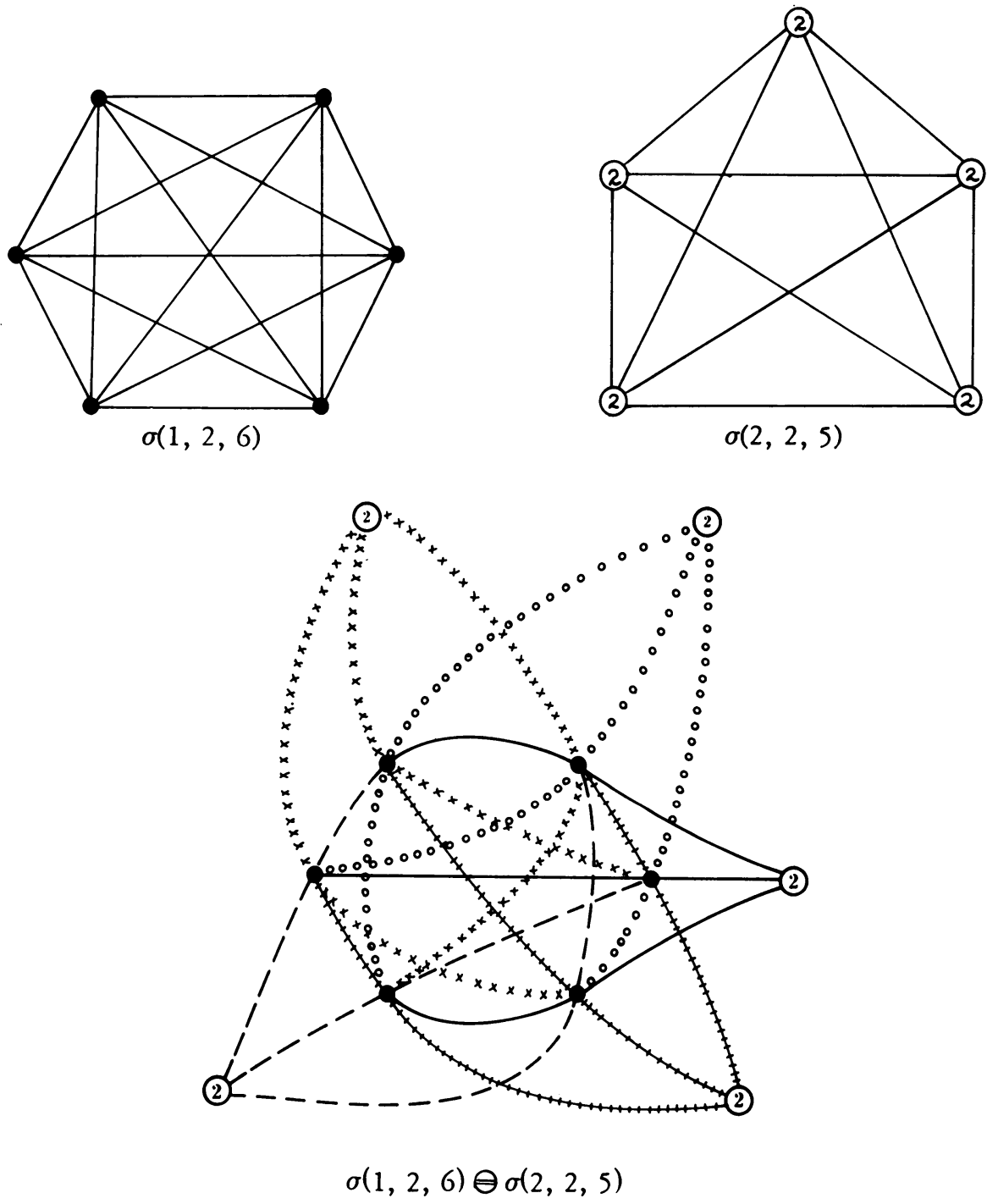

Figure 4. The complete graph extension of order 3

(The elementary lines in $\sigma(1,2,6) \ominus \sigma(2,2,5)$ joining each pair of 2-points have here been omitted for simplicity.) 
every point $a$ of $M$, and the points of $M_{a}$ are its hyperplanes. Let $\delta=k\left(M_{a}\right)$. Since the points of $M_{a}$ are disjoint, $\delta \mid c^{*}\left(M_{a}\right)=p q$, and hence $\delta=1, p, q$, or $p q$.

(80) If $\delta=1, M_{a}$ is a $\sigma(1,1, p q+1)$;

if $\delta=p, M_{a}$ is a $\sigma(p, 1, q+1)$;

if $\delta=q, M_{a}$ is a $\sigma(q, 1, p+1)$;

if $\delta=p q, M_{a}$ is a $\sigma(p q, 1,2)$.

(81) Now $M_{a}$ is not a $\sigma(p q, 1,2)$, because $\sigma(p q, 1,2)$ is just the union of two disjoint hyperplanes (points), and hence is not connected, which contradicts (42) and the assumption that $M$ is connected.

(82) Suppose that all points of $M$ have cardinality $a$.

If $a$ is any point of $M$, the points of $M_{a}$ are partitioned by points of $M$, and hence $\alpha \mid \delta$; i.e., $\alpha=1, p$, or $q$. If $\alpha=p$, then $\delta=p$ and hence $M_{a}$ is a $\sigma(p, 1, q+1)$ and $P\left(M_{a}\right) \subseteq P(M)$. But then, by (48), $M$ is a trivioid, contrary to hypothesis. A similar conclusion holds if $\alpha=q$. Hence $\alpha=1$, and since $M$ is nontrivial, the same argument used above shows that $M_{a}$ is not a $\sigma(1,1, p q+1)$.

(83) If $M_{a}$ is a $\sigma(p, 1, q+1)$ for some point $a$ of $M,|a|=1$, then the cardinality of every line containing $a$ is $p+1$, and there are $q+1$ such lines. Therefore $|E|=p q+p+1$. Likewise, if $M_{a}$ is a $\sigma(q, 1, p+1)$ for some point $a$, then $|E|=p q+q+1$. Since $p q+p+1 \neq p q+q+1$, it must be that either $M_{a}$ is a $\sigma(p, 1, q+1)$ for every $a \in P(M)$, or $M_{a}$ is a $\sigma(q, 1, p+1)$ for every $a \in P(M)$.

(84) If $M_{a}$ is a $\sigma(q, 1, p+1)$ for every $a \in P(M)$, then every line of $M$ contains $q+1$ points and every two (simple) points of $M$ are contained in a unique line. Therefore, since $|E|=p q+q+1, M$ is a $D_{2}(p q+q+1, q+1,1)$ and its $d$-sequence is $d(M)=(p q, q, 1)$. But $p q / q<q / 1$, so $d(M)$ does not satisfy (34) and $M$ does not exist.

(85) If $M_{a}$ is a $\sigma(p, 1, q+1)$ for all $a \in P(M)$, then, reasoning as in (84), we find that $M$ is a $D_{2}(p q+p+1, p+1,1)$ with $d$-sequence $(p q, p, 1)$. By (33), the number of lines of $M$ is $|\mathcal{H}|=t_{M}(0,2,3)=(p q+p)(p q+p+1) / p(p+1)$, which must be an integer. But

$$
\frac{(p q+p)(p q+p+1)}{p(p+1)}=\frac{(q+1)(p q+p+1)}{p+1}=\frac{(q+1) p q}{p+1}+q+1,
$$

so $(p+1)$ must divide $q(q+1)$. This is precisely statement (i).

(86) We may suppose therefore that the hypothesis of (82) does not hold, and that $M$ contains two points of unequal cardinality.

(87) Suppose that $M_{a}$ is a $\sigma(1,1, p q+1)$ for some point $a$. Since every point of $M_{a}$ contains a point of $M$, it follows that all points of $M$ except $a$ are simple. Thus $a \cup b$ is a line of $M$ for every $b \in P(M)-\{a\}$. Therefore, for $b \in P(M)-\{a\}, a$ is a point of $M_{b}$, and so we must have $|a|=p$ or $|a|=q$. Let 
us also note that under our hypotheses $|E|=p q+1+|a|$. If $|a|=q$, then the lines of $M$ all have cardinality $q+1$. Let $\overline{\mathcal{H}}_{a}$ be the set of lines not containing a. Clearly, $M^{\prime}=\left(E-a, \overline{\mathcal{H}}_{a}\right)$ is a simple rank 3 matroid design and $k\left(M^{\prime}\right)=q+1$. Therefore $M^{\prime}$ is a $D_{2}(p q+1, q+1,1)$ design and the $d$-sequence of $M^{\prime}$ is $d\left(M^{\prime}\right)=(p q-q, q, 1)$. However, $(p q-q) / q<q / 1$, contradicting (34).

Hence $|a|=p$. A similar argument to the above shows then that $M^{\prime}=\left(E-a, \overline{\mathcal{H}}_{a}\right)$ is a $D_{2}(p q+1, p+1,1)$, and $d\left(M^{\prime}\right)=(p(q-1), p, 1)$. In particular,

$$
t_{M^{\prime}}(0,2,3)=\frac{[p(q-1)+p][p(q-1)+p+1]}{p(p+1)}=q^{2}-\frac{q(q-1)}{p+1}
$$

must be an integer, hence $(p+1) \mid q(q+1)$. Since $a \cup b$ is a line of $M$ for every $b \in P\left(M_{a}\right), M$ is a $D_{2}(p q+1, p+1,1) \oplus P(p)$, which is (ii).

(88) By (86) and (87), we may therefore suppose that $M$ contains two points of unequal cardinality, and that for every point $a$ of $M, M_{a}$ is a $\sigma(p, 1, q+1)$ or a $\sigma(q, 1, p+1)$. If $M_{a}$ is a $\sigma(p, 1, q+1)$ then $|H-a|=p$ for every line $H$ containing $a$, and hence $|a|=k-p$. Conversely, if $|a|=k-p$, then clearly $M_{a}$ is not a $\sigma(q, 1, p+1)$, so $M_{a}$ is a $\sigma(p, 1, q+1)$. Thus $M_{a}$ is a $\sigma(p, 1, q+1)$ if and only if $|a|=k-p$, and, similarly, $M_{a}$ is a $\sigma(q, 1, p+1)$ if and only if $|a|=k-q$. In particular, every point has cardinality $k-p$ or $k-q$, and each of these cardinalities is assumed by some point.

(89) By (67) and (88), to each line $H$ of $M$ there corresponds a solution $\left(\alpha^{H}, \beta^{H}\right)$ to the diophantine equation

$$
(k-q) \alpha^{H}+(k-p) \beta^{H}=k,
$$

where $\alpha^{H}$ is the number of $(k-q)$-points in $H$ and $\beta^{H}$ is the number of $(k-p)$ points in $H$. We shall show that $0 \leq \beta^{H} \leq 3$ for every $H \in \mathcal{H}$.

(91) By (88), $|a|=k-q$ for some point $a$; hence $k \geq q+1$.

(92) Suppose that $\beta^{H} \geq 4$ for some $H \in \mathcal{H}$. If $\alpha^{H}=0$, then by (90),

$$
\beta^{H}(k-p)=k=p+(k-p) \text {, and }\left(\beta^{H}-1\right)(k-p)=p \text {, }
$$

which is impossible, since $\beta^{H}-1>1$ and $k-p \geq q+1-p>1$. Therefore $\alpha^{H} \geq 1$. Thus we have

$$
k=\alpha^{H}(k-q)+\beta^{H}(k-p) \geq 1+4(k-p),
$$

whence $4 p \geq 3 k+1$, and, using (91), $p \geq 3 q / 4+1$, which implies $p>3 q / 4+1$, since $q$ is a prime. This contradicts our hypotheses on $p$ and $q$, and proves that

$$
0 \leq \beta^{H} \leq 3 \text { for every } H \in \mathcal{H} \text {. }
$$


(94) Suppose now that $k>q+1$, and we shall derive a contradiction.

(95) If there exists a line $H$ such that $\beta^{H}=0$, then from (90) we have that $\left(a^{H}-1\right)(k-q)=q$. Therefore we must have $\alpha^{H}=2$ and $k=2 q$. By (88), let $a$ and $b$ be a $(k-q)$-point and $(k-p)$-point of $M$, respectively. Then $|a|=q,|b|=$ $2 q-p$, and the line $H^{\prime}=\operatorname{cl}(a \cup b)$ has cardinality $\left|H^{\prime}\right| \geq 2 q-p+q>2 q=k$, a contradiction. Therefore under hypothesis (94), $\beta^{H} \geq 1$ for every $H \in \mathcal{H}$.

(96) If $\beta^{H}=1$ for some line $H$ of $M$, then (90) implies that $\alpha^{H}(k-q)=p$. Hence $k=p+q$ and $a^{H}=1$. But then, by (88), every point of $M$ is a $p$-point or a $q$-point, and therefore $k=p+q$ implies that every line of $M$ is of the form $a \cup b$, where $a$ is a $p$-point, $b$ is a $q$-point. Since $M$ contains at least three points, it contains two distinct points $c$ and $c^{\prime}$ of equal cardinality, and hence the line $\mathrm{cl}\left(c \cup c^{\prime}\right)$ is not of the above form. Thus we have a contradiction.

(97) We may therefore assume that, if $k>q+1$, then $\beta^{H}=2$ or $\beta^{H}=3$ for every line $H$ of $M$. Suppose that $\left(\alpha^{H}, \beta^{H}\right)$ and $\left(\alpha^{H^{\prime}}, \beta^{H^{\prime}}\right)$ represent two distinct solutions to (90). Clearly, both $\alpha^{H^{\prime}} \neq \alpha^{H}$ and $\beta^{H^{\prime}} \neq \beta^{H}$. Let us assume then that $\beta^{H}=2$ and $\beta^{H^{\prime}}=3$. Then

$$
\alpha^{H}(k-q)+2(k-p)=k \text {, and } \alpha^{H^{\prime}}(k-q)+3(k-p)=k,
$$

whence $\left(2 \alpha^{H}-\alpha^{H^{\prime}}\right)(k-q)=p$. Since $k>q+1$, we must have $k-q=p$, which leads to a contradiction just as in (96). Therefore the solution $\left(\alpha^{H}, \beta^{H}\right)$ to (90) is the same for every $H \in \mathcal{H}$, and either $\beta^{H}=2$ or $\beta^{H}=3$. If $\beta^{H}=2$, every line $H$ contains exactly two $(k-p)$-points. Let $P_{k-p}(M)$ be the set of $(k-p)$-points of $M$, and let $a$ be a $(k-q)$-point. Then $M_{a}$ is a $\sigma(q, 1, p+1),\left|P\left(M_{a}\right)\right|=p+1$, there are exactly $p+1$ lines $H$ containing $a$, and the sets $H-a$ partition $E-a$. Hence $\left|P_{k-p}(M)\right|=2(p+1)$. On the other hand, if $b$ is a $(k-p)$-point, then $M_{b}$ is a $\sigma(p, 1, q+1)$, and so the $q+1$ lines containing $b$ each contain one $(k-p)$ point other than $b$. Hence $\left|P_{k-p}(M)\right|=q+2$. Thus $2(p+1)=q+2$, so $2 p=q$, which is impossible. If $\beta^{H}=3$, a similar argument shows that $2(q+1)+1=$ $3(p+1)$, whence $2 q=3 p$. Since $p$ and $q$ are primes, this expression implies that $p=2$ and $q=3$. However, in that case (90) becomes $a^{H}(k-3)+3(k-2)=k$, or equivalently, $\left(a^{H}+2\right)(k-3)=0$ and thus $k=3$. But then $k-q=0$, which contradicts (94).

(98) It follows that $k=q+1$.

(99) By (88) and (98), $M$ contains only simple points and $u$-points, where $u=q-p+1$. Further, $M_{a}$ is a $\sigma(q, 1, p+1)$ for every simple point $a$, so every simple point is contained in exactly $p+1$ lines of $M$. Similarly, every $u$-point is contained in exactly $q+1$ lines of $M$.

(100) Suppose that some line $H$ contains only simple points. Then $H$ contains $q+1$ points. Let $a \in P(H)$, and let $H^{\prime}$ be a line distinct from $H$ containing $a$. Because $\left|H^{\prime}-a\right|=q$ and $u \nmid q, H^{\prime}-a$ must contain a simple point $a^{\prime}$. For each 
$b \in P(H)$, there is a unique line containing $b$ and $a^{\prime}$. Hence altogether $a^{\prime}$ is contained in at least $q+1$ distinct lines, contradicting (99).

(101) By (99) and (100) it follows that every line of $M$ contains at least one $u$-point.

Now let us consider the special case $q=2 p-1$. In this case $k=q+1=2 p$ and $u=p$. For every line $H,\left(\alpha^{H}, \beta^{H}\right)$ therefore satisfies

$$
\alpha^{H}+\beta^{H} p=2 p, \quad \beta^{H} \geq 1 \text {. }
$$

It is easy to check that $\left(\alpha^{H}, \beta^{H}\right)=(0,2)$ and $\left(\alpha^{H}, \beta^{H}\right)=(p, 1)$ are the only nonnegative integral solutions to (102); i.e., every line of $M$ consists of either two $p$-points, or one $p$-point and $p$ simple points. Since every simple point $a$ is contained in exactly $p+1$. lines, and since each of these lines contains exactly one $p$-point, $M$ contains exactly $(p+1) p$-points. Let $P_{1}(M)$ be the set simple points of $M, P_{p}(M)$ the set of $p$-points of $M$, and let $A=\bigcup_{a \in P_{1}(M)} a, \mathcal{H}_{A}=$ $\left\{H \cap A: H \in \mathcal{H}\right.$ and $\left.\left(\alpha^{H}, \beta^{H}\right)=(p, 1)\right\}$. Every two distinct elements $x, y \in A$ are contained in a unique member of $\mathcal{H}_{A}$, namely $\mathrm{cl}\{x, y\}-b$, where $b$ is the unique $p$-point contained in $\mathrm{cl}\{x, y\}$. Further, every member of $\mathcal{H}_{A}$ has cardinality $k-p=p$. Finally,

$$
|A|=|E|-p\left|P_{p}(M)\right|=k+p q-p(p+1)=2 p+p(2 p-1)-p^{2}-p=p^{2} .
$$

Therefore $\left(A, \mathcal{H}_{A}\right)$ is a rank 3 PMD with $d$-sequence $(p(p-1), p-1,1)$, and so $\left(A, \mathcal{H}_{A}\right)$ is an $\operatorname{EG}(2, p)$, by (37). Since $k=2 p$, the union of every two distinct $p$-points is a line of $M$. Thus, where $B=\bigcup_{b \in P_{p}(M)} b$, and $\mathcal{H}_{B}$ is the set of lines of $M$ contained in $B,\left(B, \mathcal{H}_{B}\right)$ is a $\sigma(p, 2, p+1)$. Finally, the set of lines containing any fixed $p$-point of $M$ and meeting $A$ forms a parallel class of $\left(A, \mathcal{H}_{A}\right)$ when restricted to $A$. It follows that $M$ is an $\operatorname{EG}(2, p) \ominus \sigma(p, 2, p+1)$. This is precisely case (iii).

(103) It remains to show that if $q \neq 2 p-1$ and (98), (99), and (101) are satisfied, $M$ cannot exist.

(104) Every line of $M$ contains a simple point. Suppose to the contrary that some line is a union of $u$-points. Then $u \mid k$; that is $(q-p+1) \mid(q+1)$. But then $(q-p+1) \mid p$, which implies that $q-p+1=p$ (since $q>p)$, and hence $q=2 p-1$, contrary to our current hypothesis.

(105) Every line of $M$ contains at least two $u$-points. Indeed, by (101), every line of $M$ contains at least one $u$-point. Suppose that $H_{0}$ is a line containing exactly one $u$-point. Then $H_{0}$ contains $p$ simple points and $p+1$ points in all. Let $E^{\prime}=E-H_{0}$, and $\mathcal{H}^{\prime}=\left\{H \in \mathcal{H}: H \subseteq E^{\prime}\right\}$. For a given simple point a contained in $E^{\prime}$ and each point $b$ in $H_{0}$ there is a unique line containing $a$ and $b$, and it intersects $H_{0}$ only in $b$. Hence there are $p+1$ such lines. But $a$ is contained in a total of $p+1$ lines; hence every line containing a meets $H_{0}$. Therefore, 
every line $H^{\prime} \in \mathcal{H}^{\prime}$ is a union of $u$-points, which we have shown in (104) to be impossible. Thus $\mathcal{H}^{\prime}$ must be empty. However, let $a$ be a simple point in $H_{0}$ and let $H_{1}$ be a line distinct from $H_{0}$ containing $a$. Then, $H_{1}$ contains a $u$-point $b$, $b \subseteq E^{\prime}$, and there are exactly $(p+1)$ lines containing $b$ and meeting $H_{0}$. Hence, by (99), exactly $(q+1)-(p+1)=q-p \geq 1$ lines of $\mathcal{H}^{\prime}$ contain $b$, so $\mathcal{H}^{\prime}$ is nonempty, a contradiction.

(106) (93) and (105) together imply that, for every line $H, \beta^{H}=2$ or $\beta^{H}=3$. Using the fact that $k=q+1$, we obtain, from (90),

$$
\begin{aligned}
& \beta^{H}=2 \text { implies } \alpha^{H}=2 p-q-1 \text { for every } H \in \mathcal{H}, \text { and } \\
& \beta^{H}=3 \text { implies } \alpha^{H}=3 p-2 q-2, \text { for every } H \in \mathcal{H} .
\end{aligned}
$$

Let $\mathcal{H}_{1}$ be the set of lines (type-1 lines) containing $(2 p-q-1)$ simple points and $2 u$-points, and let $\mathcal{H}_{2}$ be the set of lines (type-2 lines) containing $3 p-2 q-2$ simple points and $3 u$-points.

(107) Let $x$ be the number of $u$-points in $M$, and let $r_{i}$ be the number of type-i lines containing any simple point of $M, i=1,2$. We must show that $r_{1}$ and $r_{2}$ are well defined. This follows by observing that $r_{1}+r_{2}=p+1$, and $r_{1}=3(p+1)-x$. The last relationship follows from the fact that every simple point $a$ lies on $(p+1)$ lines, each containing at most three and at least two $u$-points. Hence $3(p+1) \geq x \geq 2(p+1)$, and $3(p+1)-x$ is the number of lines containing $a$ with precisely two $u$-points.

(108) Let $R_{i}$ be the number of type-i lines containing any $u$-point, $i=1,2$. Reasoning as in (107), we find that

$$
R_{1}+R_{2}=(q+1) \text { and } R_{1}=(2(q+1)+1)-x
$$

and hence $R_{1}$ and $R_{2}$ are well defined.

(109) Letting $y$ denote the number of simple points in $M$, we have $(q-p+1) x+y=p q+q+1=|E|$, where $x>0$ and $y>0$ by assumption.

(110) Let $b_{i}$ denote the number of type-i lines in $M, i=1,2$. Since every type-1 line contains $2 p-q-1$ simple points, and since every simple point is contained in $r_{1}$ type-1 lines, we have, using (107) and (109),

$$
(2 p-q-1) b_{1}=r_{1} y=(3(p+1)-x)(p q+q+1-(q-p+1) x) .
$$

Using similar arguments we obtain the following equations.

$$
\begin{aligned}
2 b_{1} & =R_{1} x=[2(q+1)+1-x] x, \\
(3 p-2 q-2) b_{2} & =r_{2} y=[x-2(p+1)][p q+q+1-(q-p+1) x], \\
3 b_{2} & =R_{2} x=[x-(q+2)] x .
\end{aligned}
$$

These four equations in $b_{1}, b_{2}$, and $x$ must have a positive integral solution for $M$ to exist. The equations are consistent, as may be verified by eliminating $b_{1}$ and $b_{2}$. 
(115) Suppose that for some solution to this system, $b_{2}=0$. Then $x=q+2$, by (114) and (109). Substituting $b_{2}=0$ and $x=q+2$ in (113) we have that either

$$
q+2-2(p+1)=0 \text { or }(p q+q+1)-(q-p+1)(q+2)=0 .
$$

The first implies that $2 p=q$, which is absurd. The second yields

$$
\begin{aligned}
p q+q+1 & =q^{2}-p q+3 q-2 p+2, \\
2 p q+2 p & =q^{2}+2 q+1, \quad 2 p(q+1)=(q+1)^{2},
\end{aligned}
$$

so $2 p=q+1$, which is contrary to our present hypothesis (103).

Therefore we may assume that $b_{2}>0$. Further, $r_{2}>0$ and $y>0$, so (113) implies that $3 p-2 q-2>0$, and hence $p \geq 2 q / 3+1$.

Eliminating $b_{1}$ from (111) and (112), we find that $x$ must be a positive integral root of the quadratic $\theta_{p, q}(x)$ :

However, since $p \geq 2 q / 3+1, \theta_{p, q}(x)$ has no positive integral root. Hence the equations (111)-(114) have no positive integral solution, and $M$ cannot exist. Thus (103) is proved, and likewise the theorem.

The proof of (78) is valid for any pair of distinct primes $p$ and $q$ for which $p \leq 3 q / 4+1$ and the associated equations (111)-(114) have no positive integral solution in $x$. The pairs $(p, q)$ as above for which such solutions do exist, or even those pairs for which only $\Theta_{p, q}(x)$ has a positive integral root, appear to be very rare. In fact, the pair $(17,23)$ is the only one known to this author. The existence of a rank 3 , nontrivial matroid design with cocircuit cardinality $17 \cdot 23=391$ is unknown at this time. (If such a matroid does exist, the structural information about it obtainable from the above proof shows that it is closely related to an affine plane of order 10 , and probably at least as hard to construct.)

In the statement of (78), if the prime $p$ satisfies $2 q / 3+1 \leq p<3 q / 4+1$ then it is straightforward to show that none of the conditions (i), (ii), (iii) can hold. Thus, using (60), we have the following.

(117) Corollary. If $p$ and $q$ are distinct primes such that $2 q / 3+1 \leq p \leq 3 q / 4+1$ and $\theta_{p, q}(x)$ bas no positive integral root, then every connected matroid design with cocircuit cardinality $p q$ is trivial.

If, on the other hand, $p<2 q / 3+1$ and $(p, q) \neq(2,3),(p, q) \neq(3,5)$, then it may easily be shown that at most one of the conditions (i), (ii), (iii) of (78) can hold. Moreover, under these assumptions $p+1$ must be relatively prime to $q$, and hence the conditions (i), (ii), (iii) may be simplified to yield the following result.

(118) Corollary. Let $p$ and $q$ be distinct primes sucb that $p<2 q / 3+1$ and $(p, q) \neq(2,3),(3,5)$. 
(i) If $(p+1) \mid(q+1)$, then every nontrivial member of $\mathbb{M}_{3}(p q)$ is a $D_{2}(p q+p+1, p+1,1)$.

(ii) If $(p+1) \mid(q-1)$, then every nontrivial member of $\mathbb{M}_{3}(p q)$ is a $D_{2}(p q+1, p+1,1) \oplus P(p)$.

(iii) If $q=2 p-1$, then every nontrivial member of $\Pi_{3}(p q)$ is an $\operatorname{EG}(2, p) \ominus$ $\sigma(p, 2, p+1)$.

(iv) If $(p+1) \nmid(q+1),(p+1) \nmid(q-1)$, and $q \neq 2 p-1$, then every connected matroid design with cocircuit cardinality $p q$ is trivial.

The pair of primes $(7,11)$ satisfies (308)(iv); hence there are no nontrivial matroid designs with cocircuit cardinality 77 . The pair $(5,7)$ satisfies $(308)(\mathrm{ii})$, and thus every nontrivial member of $\pi_{3}(35)$ must be an $\operatorname{EG}(2,6) \oplus P(5)$. But there exists no $\operatorname{EG}(2,6)$. Hence by $(241)$ every matroid design with cocircuit cardinality 35 is trivial. The class of cocircuit cardinalities for which no nontrivial matroid design exists is therefore larger than the class of all primes.

Characterization of higher rank equicardinal matroids. We turn our attention now to the problem of determining $\pi_{n}(\gamma)$ when $n>3$ and $\gamma$ is the product of two primes.

(119) Theorem. (i) Where $p$ is an odd prime, every member of $\bigcup_{n=4}^{\infty} \pi_{n}\left(p^{2}\right)$ is trivial.

(ii) The only nontrivial member of $\bigcup_{n=4}^{\infty} \pi_{n}\left(2^{2}\right)$ is the rank 4 matroid EG $(3,2)$.

Proof Let $p$ be a prime (either odd or even), and let $M=(E, \mathcal{H})$ be a nontrivial member of $\pi_{4}\left(p^{2}\right)$.

(120) $M_{a}=M \cdot(E-a)$ is nontrivial for some point $a$ of $M$, and hence $M_{a}$ is a $\mathrm{PG}(2, p)$, by (69). In particular, $M_{a}$ is simple; hence $P\left(M_{a}\right) \cup\{a\}=P(M)$. By the same reasoning, $M_{b}$ is a $\mathrm{PG}(2, p)$ for some $b \in P\left(M_{a}\right)$, hence $a$ is also simple. Therefore, $M$ is simple, and

$$
|E|=\left|P\left(M_{a}\right)\right|+|a|=p^{2}+p+2 .
$$

(122) $M_{b}$ is nontrivial for every point $b$; for otherwise, by (69), $M_{b}$ would be a $\sigma\left(1,2, p^{2}+2\right)$ or a $\sigma(p, 2, p+2)$ and correspondingly $|E|=p^{2}+3$ or $|E|=p^{2}$ $+2 p+1$, contrary to $(121)$.

(123) Hence $M_{b}$ is a $P G(2, p)$ for every $b \in P(M)$. It follows that all points and lines of $M$ are simple, and $k(M)=p+2$. (Recall that a simple $k$-flat is a flat of cardinality $k_{\text {.) }}$ It follows that $M$ is a PMD with $d$-sequence $d(M)=\left(p^{2}, p, 1,1\right)$. If $p=2$, then $d(M)=(4,2,1,1)$, so $M$ is an $\operatorname{EG}(3,2)$, by (37). If $p \neq 2$, consider the number $t_{M}(0,3,4)$ of hyperplanes in $M$. Using (33),

$$
t_{M}(0,3,4)=\frac{\left(p^{2}+p\right)\left(p^{2}+p+1\right)\left(p^{2}+p+2\right)}{p(p+1)(p+2)}=\frac{\left(p^{2}+p+1\right) p^{2}}{p+2}+p^{2}+p+1 \text {. }
$$


Now, since $p>2, p^{2}$ and $p+2$ are relatively prime, so $\left(p^{2}+p+1\right) /(p+2)=$ $p-1+3 /(p+2)$ must be an integer, which is absurd. Hence, for $p \neq 2$, every member of $\mathbb{M}_{4}\left(p^{2}\right)$ is trivial, so every member of $\bigcup_{n=4}^{\infty} \Re_{n}\left(p^{2}\right)$ is trivial, and (i) is proved.

To complete the proof of (ii), we shall show that if $M=(E, \mathcal{H}) \in M_{5}\left(2^{2}\right)$, then $M$ is trivial. If $M$ is nontrivial, then by (123) $M_{a}=M \cdot(E-a)$ is an $E G(3,2)$ for some point $a$ of $M$. Using the fact that an $\operatorname{EG}(3,2)$ is simple, an argument similar to that of (120)-(122) shows that $M_{a}$ is an $\operatorname{EG}(3,2)$ for every point $a$ of $M$. It follows that $M$ is a PMD with $d$-sequence $(4,2,1,1,1)$. But then,

$$
t_{M}(0,4,5)=\frac{(4+2)(4+2+1)(4+2+1+1)(4+2+1+1+1)}{2 \cdot 3 \cdot 4 \cdot 5}=\frac{6 \cdot 7 \cdot 8 \cdot 9}{2 \cdot 3 \cdot 4 \cdot 5},
$$

which is no integer, and therefore no such $M$ exists. Thus every member of $\pi_{5}\left(2^{2}\right)$ is trivial, which, combined with (59), completes the proof.

The determination of $\prod_{n}(p q)$ for $n \geq 4$ when $p$ and $q$ are distinct primes such that $p<2 q / 3+1$ has not been completely solved. We know, for example (see [15]), that $\mathbb{M}_{n}(6)$ contains nontrivial matroid designs for $3 \leq n \leq 18$. Likewise $\mathbb{M}_{n}(15)$ contains a large variety of structures for $n \geq 4$. Excluding these cases, however, we can state some rather general results. In what follows, we shall treat the various cases corresponding to (i), (ii), and (iii) of (118) separately.

(124) Theorem. If $p$ and $q$ are distinct primes sucb that $q=2 p-1$ and $(p, q) \neq(2,3),(3,5)$, then every member of $\bigcup_{n=4}^{\infty} \pi_{n}(p q)$ is trivial.

Proof. It suffices to prove that every member of $\Re_{4}(p q)$ is trivial. Let $M=(E, H) \in \mathbb{M}_{4}(p q)$ be nontrivial; then by (53) and (118) there is a point a such that $M_{a}$ is an $\operatorname{EG}(2, p) \ominus \sigma(p, 2, p+1)$. The simple points of $M_{a}$ are simple points of $M$, and there are more than two of them, so $M_{b}$ is an $\operatorname{EG}(2, p) \ominus$ $\sigma(p, 2, p+1)$ for some simple point $b$. Thus

$$
|E|=2 p^{2}+p+1 \text {. }
$$

Let $d_{1}, d_{2}, \cdots, d_{p+1}$ be the $p+1$-points of $M_{b} . L_{i}=d_{i} \cup b$ is then a line of $M$ for each $i, 1 \leq i \leq p+1$. Suppose that for some $i, d_{i}$ is also a point of M. Since $\left|E-d_{i}\right|=2 p^{2}+1, M_{d_{i}}$ is not an $\operatorname{EG}(2, p) \ominus \sigma(p, 2, p+1)$, so, $M_{d_{i}}$ must be trivial. Since $L_{i}-d_{i}$ is a simple point of $M_{d_{i}}, M_{d_{i}}$ must be a $\sigma(1,2, p q+2)$, which contradicts the fact that $\left|E-d_{i}\right|^{2}=2 p^{2}+1$. Therefore $d_{i}$ is not a point of $M$ for $1 \leq i \leq p+1$, and each line $L_{i}$ contains three distinct points of $M: b, e_{1}^{i}, e_{2}^{i}$.

Suppose now that $M_{c}$ is trivial for some point $c$. Then the union of every two lines of $M$ containing $c$ is a hyperplane of $M$. Let $L_{i}$ be distinct from $\operatorname{cl}(b \cup c)$. Then $H=\operatorname{cl}\left(c \cup e_{1}^{i}\right) \cup \operatorname{cl}\left(c \cup e_{2}^{i}\right)$ is a hyperplane of $M$ disjoint from $b$; but $\left.b \subseteq L_{i}=\mathrm{cl}\left\{e_{1}^{i}, e_{2}^{i}\right\}\right) \subseteq H$, a contradiction. 
Therefore for every $c \in P(M), M_{c}$ is an $\operatorname{EG}(2, p) \ominus \sigma(p, 2, p+1)$. In particular, for every $c \in P(M),|c|=|E|-\left(2 p^{2}+p\right)=1$, by (125); hence $M$ is simple.

Now an EG $(2, p)$ contains exactly $p^{2}+p$ lines, and a $\sigma(p, 2, p+1)$ contains $\left(p^{2}+p\right) / 2$ lines. Thus, by construction, an $\mathrm{EG}(2, p) \ominus \sigma(p, 2, p+1)$ contains exactly $p^{2}+p+\left(p^{2}+p\right) / 2=3\left(p^{2}+p\right) / 2$ lines, and further, each line has cardinality $2 p$. Therefore every point of $M$ is contained in exactly $3\left(p^{2}+p\right) / 2$ hyperplanes of cardinality $2 p+1$, so

$$
|\mathfrak{H}|=\frac{3\left(p^{2}+p\right)}{2} \cdot \frac{\left(2 p^{2}+p+1\right)}{2 p+1}=\frac{3 p\left(p^{2}+p\right)}{2}+\frac{3\left(p^{2}+p\right)}{2(2 p+1)}
$$

Since $3 p\left(p^{2}+p\right) / 2$ is an integer, $2(2 p+1) \mid 3\left(p^{2}+p\right)$, and since $p$ is a prime and $p \neq 2$ by hypothesis, we must have $2(2 p+1) \mid 3(p+1)$. Thus $4 p+2 \leq 3 p+3$, which is absurd. Therefore $\mathbb{M}_{4}(p q)$, and hence $\bigcup_{n=4}^{\infty} \Re_{n}(p q)$, contains only trivioids.

Preparatory to considering $\bigcup_{n=4}^{\infty} \pi_{n}(p q)$ for cases (i) and (ii) of (118), we need to introduce the following general concept. For any matroid $M$, the independence number of $M, \beta(M)$, is the largest integer $k$ for which every $k$-subset in $M$ is independent. Thus $\beta(M) \geq 2$ is equivalent to the statement that $M$ is simple.

(126) Theorem. If, for some integers $\gamma \geq 1, n \geq 3$, and $m \geq 2$, every nontrivial $M \in \mathbb{M}_{n}(\gamma)$ satisfies $\beta(M) \geq m$, then for every integer $n^{\prime} \geq n$ and every nontrivial $\left.M^{\prime} \in\right)_{n^{\prime}}^{n}(\gamma), \beta\left(M^{\prime}\right) \geq m+\left(n^{\prime}-n\right)$.

Proof. First, if every member of $\pi_{n}(\gamma)$ is trivial, then every member of $\mathbb{M}_{n^{\prime}}(\gamma)$ is trivial for $n^{\prime} \geq n$, by (59), and the theorem is vacuously true. Second, let us observe that for any matroid $M, \beta(M) \leq r(M)$; hence $m \leq n$. We shall prove that if (126) holds for $n^{\prime} \geq n$, it holds for $n^{\prime}+1$. (Clearly it holds if $n^{\prime}=n$.) Let $M=(E, \mathcal{H}) \in \mathbb{M}_{n^{\prime}+1}(\gamma)$ be nontrivial. $M$ is simple, because otherwise we could find a point $a$ of $M$ such that $M_{a}$ is both nontrivial and nonsimple, i.e., such that $\beta\left(M_{a}\right)=1$, which contradicts the induction hypothesis. Further, every line of $M$ is simple. If not, let $L$ be a line containing three distinct points $a_{1}, a_{2}, a_{3}$. Since $L-a_{i}$ is a point of $M_{a_{i}}$ for each $i, M_{a_{i}}$ is nonsimple, hence a trivioid. But then $M$ is a trivioid, contrary to hypothesis.

Now, let $S$ be any subset of $E$ having cardinality $m+\left(n^{\prime}+1\right)-n$ and let $x \in S$. If $M_{\{x\}}$ is nontrivial, then by the induction hypothesis the $\left(m+n^{\prime}-n\right)$-subset $S-\{x\}$ is independent in $M_{\{x\}}$, hence $S$ is independent in $M$. If $M_{\{x\}}$ is trivial, then, since every line of $M$ is simple, it must be the simple trivioid $\sigma(1, k(M)-1,|E|-1)$. Since $r\left(M_{\{x\}}\right)=n^{\prime}$, every $n^{\prime}$-subset of $E-\{x\}$ is independent in $M_{\{x\}}$. Since $m \leq n$, it follows that the $\left(m+n^{\prime}-n\right)$-subset $S-\{x\}$ is independent in $M_{\{x\}}$, from which it follows that $S$ is independent in $M$. 
(127) Theorem. If $p$ and $q$ are distinct primes such that $(p+1) \mid(q+1)$, then, for $n \geq 3$, every nontrivial member of $\mathbb{M}_{n}(p q)$ is a $D_{n-1}(p q+p+n-2, p+n-2,1)$.

Proof. If $p$ and $q$ are distinct primes such that $(p+1) \mid(q+1)$, then certainly $p<2 q / 3+1$, and, further, $(p, q) \neq(2,3),(3,5)$.

Hence, by $(118)(\mathrm{i})$, every nontrivial member of $\mathbb{M}_{3}(p q)$ is a $D_{2}(p q+p+1, p+1,1)$, and is therefore simple. With $m=2$ and $n=3$ in (126) it follows that $\beta(M) \geq n^{\prime}-1$ for every nontrivial $M \in \pi_{n^{\prime}}(p q)$. But then every such $M$ is a PMD with $d$-sequence of form $(p q, \alpha, 1, \ldots, 1)$, a sequence of $n^{\prime}$ terms.

Thus by (34) we must have $p q / \alpha \geq \alpha / 1$ and $\alpha \mid p q$, so $\alpha=p$. Thus every such $M$ has $d$-sequence $(p q, p, 1, \ldots, 1)$, so by $(36), M$ is an $\left(n^{\prime}-1\right)-\left(p q+p+n^{\prime}-2, p+n^{\prime}-2,1\right)$ design. $\square$

In the final case, where $p$ and $q$ are distinct primes satisfying $p<2 q / 3+1$ and $(p+1) \mid(q-1)$, there is a great variety of structures in $\mathbb{M}_{n}(p q)$ for $n \geq 4$. We shall, therefore, restrict our attention to the analysis of $\pi_{4}(p q)$ in this instance.

As a preliminary, we are going to introduce a generalization of $t$-designs that has a considerable theoretical interest in its own right.

Let $\mathcal{S}$ be a collection of nonempty subsets partitioning a finite set $E$. A subset $T$ of $E$ such that $|T \cap S| \leq 1$ for every $S \in \mathcal{S}$ is said to be a partial transversal (PT) of $\mathcal{S}$, and $|T|$ is its length.

A triple $(E, \mathcal{S}, \mathcal{T})$ is a transversal design with parameters $t, v, s, k, \lambda$, where $v \geq k \geq t \geq 2, s \geq 1, \lambda \geq 1$, if

(i) $\mathcal{S}$ is a family of $v$ nonempty disjoint subsets that partition the finite set $E$ and $|S|=s$ for every $S \in \mathcal{S}$,

(ii) $\mathcal{T}$ is a family of PT's of $\mathcal{S}$ such that $|T|=k$ for every $T \in \mathcal{T}$;

(iii) every PT of length $t$ is contained in exactly $\lambda$ members of $\mathcal{T}$. For short we refer to any such triple $(E, \mathcal{S}, \mathcal{J})$ as a $\operatorname{TD}_{t}(\nu, s, k, \lambda)$, and to any transversal design in general as a TD.

Certain subclasses of transversal designs have played an important role in the construction of BIBD's. TD's with $t=2, v=k$, and $\lambda=1$ have been called $T$-systems by Hanani [7]; and systems with $t=2$ are known more generally as group divisible designs. Also, as we shall presently show, the existence of TD's with $v=k$ is equivalent to the existence of so-called orthogonal arrays. Thus, transversal designs generalize these various notions in much the same way that $t$-designs generalize the concept of BIBD's.

The application of transversal designs to the construction of matroid designs is as follows.

(128) Theorem. Let $(E, \mathcal{S}, \mathcal{J})$ be a $\operatorname{TD}_{3}(\nu, s, s+1,1)$ for some integer $s \geq 2$, and let $\mathcal{H}$ be the family $\mathcal{T} \cup\{S \cup\{x\}: S \in \mathcal{S}$ and $x \in E-S\}$ of $(s+1)$-subsets of $E$. Then $(E, \mathcal{H})$ is a simple rank 4 matroid design, and, if $s \geq 3,(E, \mathcal{H})$ is not a PMD. 
Proof. (129) Where $H_{1}$ and $H_{2}$ are distinct members of $\mathcal{H}$, clearly either $H_{1} \cap H_{2}=S_{i}$ for some $i$, or else $H_{1} \cap H_{2}$ is a PT of length $\leq 2$ (possibly empty). Conversely, since $s \geq 2$, every $S_{i}$ may be written as $S_{i}=\left(S_{i} \cup\{x\}\right) \cap$ $\left(S_{i} \cup\{y\}\right)$, where $x$ and $y$ are distinct members of $S_{j}$ for some $j \neq i$. Likewise, every $\mathrm{PT}\{x, y\}$ of $\mathcal{S}$ of length 2 , where $x \in S_{i}$ and $y \in S_{i}$, may be written as $\{x, y\}=\left(S_{i} \cup\{y\}\right) \cap\left(S_{j} \cup\{x\}\right)$. Thus every member of $\mathcal{\complement}=\mathcal{T}_{2} \cup \mathcal{S}$, where $\mathcal{T}_{2}$ is the set of PT's of length 2, is representable as the intersection of members of $\mathcal{H}$; moreover, every such nonempty intersection is either a singleton or an element of . We shall show that for every $L \in \mathfrak{Q}$ and $x \in E-L, L \cup\{x\}$ is contained in a member of $\mathcal{H}$, and it will follow from the preceding remarks that $M$ is a simple rank 4 matroid design with line-family $\mathcal{Q}$ and hyperplane-family $\mathcal{H}$. Further, $M$ will be a PMD if and only if the lines are equicardinal, that is, if and only if $s=2$.

(130) Let $L \in \mathfrak{Q}$ and $x \in E-L$. If $L=S_{i}$ for some $i$, then $L \cup\{x\} \in \mathcal{H}$ by construction. Otherwise, $L=\{a, b\}$ for some $a \in S_{i}$ and $b \in S_{j}$, where $i \neq j$. If $x \in S_{j}$, then $L \cup\{x\} \subseteq S_{j} \cup\{a\} \in \mathcal{H}$, and a similar result holds if $x \in S_{i}$. Otherwise, $x \in S_{k}$ for some index $k$ distinct from $i$ and $j$. Then $\{a, b, x\}$ is a partial transversal of length 3 , hence it is contained in a unique member of $\mathfrak{T} \subseteq \mathcal{H}$.

Any matroid $(E, \mathcal{H})$ constructed as above from a $\operatorname{TD}_{3}(v, s, s+1,1)$ will be called a $\operatorname{TMD}(v, s)$ (transversal matroid design with parameters $v, s$ ). The hyperplane cardinality of any $\operatorname{TMD}(v, s)$ is $s+1$ and the cocircuit cardinality is $(v-1) s-1$.

(131) Theorem. If $p$ and $q$ are distinct primes sucb that $p<2 q / 3+1$ and $(p+1) \mid(q-1)$ but $(p, q) \neq(2,3),(p, q) \neq(3,5)$, then every nontrivial member of $\mathbb{M}_{4}(p q)$ is either (i) a $D_{3}(p q+2, p+2,1) \oplus P(p)$; or (ii) $a \operatorname{TMD}(q+1-(q-1) /(p+1), p+1)$.

Proof. Let $M=(E, \mathcal{H}) \in \mathbb{M}_{4}(p q)$ be nontrivial.

Suppose first $M$ is not simple, and let $a \in P(M),|a|>1$.

By (53) and (118), there is a point $b \neq a$ such that $M_{b}$ is a $D_{2}(p q+1, p+1,1) \oplus P(p)$, which we abbreviate for this discussion by $D_{2}(p)$. Evidently, $\operatorname{cl}_{M}(a \cup b)-b$ is the unique nonsimple point of $M_{b}$, hence every point in $P(M)-P\left(\mathrm{cl}_{M}(a \cup b)\right)$ must be simple. There are at least two such points; hence there is a simple point $c \in P(M)-P\left(\operatorname{cl}_{M}(a \cup b)\right)$ such that $M_{c}$ is a $D_{2}(p)$. Then, as above, we conclude that every point in $P(M)-P\left(\operatorname{cl}_{M}(a \cup c)\right)$ is simple, and so $a$ is the only nonsimple point of $M$. Moreover, since $|a| \neq|b|, M_{a}$ and $M_{b}$ are nonisomorphic matroids. Thus, $M_{a}$ is not a $D_{2}(p)$, so, by (118) $M_{a}$ is a trivioid.

Let $a$ be the common cardinality of the points of $M_{a}$. Then $a \mid c^{*}\left(M_{a}\right)=p q$; hence $a=1, p, q$, or $p q$. Suppose that $\alpha \geq p$, and let $L$ be a line of $M$ containing $a$. Then $|L-a|=a \geq p \geq 2$. Since the points of $M$ in $L-a$ are simple, $M_{d}$ is nontrivial, hence a $D_{2}(p)$, for some simple point $d$ in $L-a$. But $L-d$ is a point of $M_{d}$, and $|L-d|>p$, which is impossible because a $D_{2}(p)$ contains no 
$m$-point for $m>p$. Hence $\alpha=1$ and $M_{a}$ must be a $\sigma(1,2, p q+2)$.

Since $M_{d}$ is a $D_{2}(p),|E|=p q+p+1+|d|=p q+p+2$; whereas from $M_{a}=\sigma(1,2, p q+2)$ we have that $|E|=p q+|a|+2$. Thus $|a|=p$. Further, $k(M)=p+2$.

Let $\mathcal{H}^{\prime}$ be the family of hyperplanes and $\mathcal{L}^{\prime}$ be the family of lines of $M$ contained in $E-a$. For any distinct elements $x, y \in E-a, L=\operatorname{cl}_{M}(\{x, y\}) \in \mathcal{Q}^{\prime}$, for otherwise $a \subseteq L$, and hence $|L| \geq p+2=k(M)$, which is absurd, because $L$ is properly contained in some hyperplane of $M$. For any $L \in \mathfrak{Q}^{\prime}, \operatorname{cl}_{M}(L \cup a) \in \mathcal{H}$, hence $\left|\mathrm{cl}_{M}(L \cup a)\right|=p+2$. Thus, $|L|=2$, i.e., every member of $\mathcal{L}^{\prime}$ is simple. For any $L \in \mathfrak{Q}^{\prime}$ and $x \in(E-a)-L, H=\operatorname{cl}_{M}(L \cup\{x\}) \in \mathcal{H}^{\prime}$, otherwise $|H| \geq|L \cup\{x\}|$ $+|a|=p+3>k(M)$, which is absurd.

Thus, $M^{\prime}=\left(E-a, \mathcal{H}^{\prime}\right)$ is a simple, rank 4 PMD with $d$-sequence $(p q, p, 1,1)$, so $M^{\prime}$ is a $D_{3}(p q+2, p+2,1)$. Since $M=M^{\prime} \oplus a, M$ is a $D_{3}(p q+2, p+2,1) \oplus P(p)$.

Suppose on the other hand that $M$ is simple. Then $M_{a}$ is a $D_{2}(p)$ for some $a \in P(M)$, and it follows by considering the sizes of the possible trivioids in $M_{3}(p q)$ that $M_{a}$ is a $D_{2}(p)$ for every $a \in P(M)$. We also note that $|E|=p q+p+2$ and $k(M)=p+2$.

Since a $D_{2}(p)$ contains exactly one nonsimple point, which has cardinality $p$, it follows that every point of $M$ is incident with exactly one nonsimple line, which has cardinality $p+1$. Thus $E$ is partitioned by $v=q+1-(q-1) /(p+1)$ lines of cardinality $p+1$. Let the family of these lines be denoted by $\mathcal{S}=\left\{S_{1}, S_{2}, \ldots, S_{v}\right\}$. Since $k(M)=p+2, S_{i} \cup\{x\} \in \mathcal{H}$ for any $S_{i} \in \mathcal{S}$ and $x \in E-S_{i}$. Let $\mathcal{H}^{v^{\prime}}=\left\{S_{i} \cup\{x\}: S_{i} \in \mathcal{S}\right.$ and $\left.x \in E-S_{i}\right\}$. For any $H \in \mathcal{H}-\mathcal{H}^{\prime}$, $\left|H \cap S_{i}\right| \leq 1$ for every $S_{i} \in \mathcal{S}$ (since $H$ is closed); thus, $H$ is a partial transversal of length $p+2$.

Any partial transversal $r$ of $\mathcal{S}$ having length 3 is contained in no line of $M$, hence $\operatorname{cl}(\tau) \in \mathcal{H}$, and clearly $\operatorname{cl}(\tau) \notin \mathcal{H}^{\prime}$. It follows that $\left(E, \mathcal{S}, \mathcal{H}-\mathcal{H}^{\prime}\right)$ is a $\mathrm{TD}_{3}(q+1-(q-1) /(p+1), p+1, p+2,1)$, and, by $(129), M$ is a $\operatorname{TMD}(q+1-(q-1) /(p+1), p+1)$.

The existence of transversal designs. Little appears to be known about the existence of transversal designs in general. However, enough is known about certain subclasses to establish the existence of several infinite classes of transversal designs that give rise to matroid designs.

First let us make two general observations about TD's. For a given $\mathrm{TD}_{t}(v, s, k, \lambda), D=(E, \mathcal{S}, \mathfrak{T})$, standard counting arguments may be used to show that the number of transversals in $D$ containing any length-i transversal of $\mathcal{S}$ is

$$
\lambda_{i}=\lambda\left(\begin{array}{c}
v-i \\
t-i
\end{array}\right) s^{t-i} /\left(\begin{array}{c}
k-i \\
t-i
\end{array}\right), \quad 0 \leq i \leq t .
$$

The requirement that the numbers $\lambda_{i}$ be integers generalizes the consistency conditions for BIBD's. 
Secondly, as in the case of $t$-designs, we may "compose" certain pairs of TD's, as shown in the following theorem.

(133) Theorem. If there exists a $\operatorname{TD}_{t}(v, s, k, 1)$ and a $\operatorname{TD}_{t}\left(w, s^{\prime}, v, \lambda^{\prime}\right)$, then there exists a $\operatorname{TD}_{t}\left(w, s s^{\prime}, k, \lambda^{\prime}\right)$.

Proof. Let $\left(E^{\prime}, \mathcal{S}^{\prime}, \mathcal{J}^{\prime}\right)$ be a $\operatorname{TD}_{t}\left(w, s^{\prime}, v, \lambda^{\prime}\right)$, where $\mathcal{S}^{\prime}=\left\{S_{1}^{\prime}, S_{2}^{\prime}, \ldots, S_{w}^{\prime}\right\}$, and the members of $E^{\prime}$ are chosen to be disjoint sets, each of cardinality $s$. Then each $T \in \mathfrak{T}^{\prime}$ is a collection of $v$ disjoint $s$-sets, so let $\left(\bigcup_{x \in T} x, T, \mathcal{J}_{T}\right)$ be a $\operatorname{TD}_{t}(v, s, k, 1)$. For each $i, 1 \leq i \leq w$, let $S_{i}=\bigcup_{x \in S_{i}^{\prime}} x$, and let $\mathcal{S}=\left\{S_{1}, \cdots, S_{w}\right\}$. Further, let $E=\bigcup_{x \in E^{\prime}} x$ and $\overline{\mathfrak{T}}=\bigcup_{T \in \mathfrak{T}^{\prime}} \mathfrak{T}_{T}^{i}$.

Let $A=\left\{a_{1}, \ldots, a_{t}\right\}$ be a PT of $\mathcal{S}$, where $a_{i} \in \sigma_{i} \in S_{i}^{\prime}$ for each $i, 1 \leq i \leq t$. Then $\left\{\sigma_{1}, \cdots, \sigma_{t}\right\}$ is a $\mathrm{PT}$ of $\mathcal{S}^{\prime}$ of length $t$, and hence is contained in $\lambda^{\prime}$ members of $\mathcal{J}^{\prime}$. For each $T \in \mathcal{T}^{\prime}$ containing $\left\{\sigma_{1}, \ldots, \sigma_{t}\right\}$, there is precisely one member of $\mathfrak{T}_{T}$ containing $A$, and hence altogether there are precisely $\lambda^{\prime}$ members of $\mathcal{T}$ containing $A$. Thus $(E, \mathcal{S}, \mathcal{J})$ is a $\operatorname{TD}_{t}\left(w, s s^{\prime}, k, \lambda^{\prime}\right)$.

Except for the $t$-designs, the only class of transversal designs about which much is known are those in which the transversals are full, that is, those for which $v=k$. For this case the consistency conditions of (132) are always satisfied. Let $(E, \mathcal{S}, \mathcal{J})$ be a given $\operatorname{TD}_{t}(k, s, k, 1)$, and for each $S \in \mathcal{S}$ let us number the elements $1,2, \ldots, s$ and let us index the sets in $\delta s_{1}, \ldots, s_{k}$. With each given transversal $T$ we then associate the column vector $C_{T}$ whose $i$ th element is defined to be the number of the unique $x \in S_{i} \cap T$. Any array of these $\lambda s^{t}$ column vectors is a $k \times \lambda s^{t}$ matrix $A$ with entries from $1,2, \ldots, s$ such that any $t$-rowed submatrix of $A$ lists every ordered triple from $1,2, \ldots, s$ exactly $\lambda$ times. Thus $A$ is an orthogonal array $\mathrm{OA}\left(\lambda s^{t}, k, s, t\right)$ of size $\lambda s^{t}$, order $k$, level $s$, strength $t$ and index $\lambda$.

Conversely, given an orthogonal array $\mathrm{OA}\left(\lambda s^{t}, k, s, t\right)$ its columns define the transversals of a $\operatorname{TD}_{t}(k, s, k, t)$ on $k$ disjoint $s$-sets, one for each row.

There are a variety of results concerning the existence of orthogonal arrays. A very basic one is that the existence of an $\mathrm{OA}\left(s^{2}, k, s, 2\right)$ is equivalent to the existence of $k-2$ mutually orthogonal Latin squares of order $s$ [1]. Of particular interest here is Bush's construction [2] of an $\mathrm{OA}\left(q^{3}, q+1, q, 3\right)$ for every prime power $q$. Using this fact we have the following.

(134) Theorem. For any prime power $q$, there exists a $\operatorname{TD}_{3}(q+1, q, q+1,1)$, and bence $a \operatorname{TMD}(q+1, q)$.

In particular, by (134), there exists a $\operatorname{TD}_{3}(4,3,4,1)$. Now Hanani [6] has shown that for every integer $v \equiv 2$ or $4(6)$, there exists a $3-(v, 4,1)$ design, i.e., a $\operatorname{TD}_{3}(\nu, 1,4,1)$. Thus by the composition theorem (133), we have the following. 
(135) Theorem. For any integer $v \geq 4^{-}$such that $v \equiv 2$ or 4 (6), there exists a $\operatorname{TD}_{3}(v, 3,4,1)$ and bence $a \operatorname{TMD}(\nu, 3)$.

Thus Theorems (134) and (135) identify three infinite classes of simple rank 4 matroid designs which are not perfect matroid designs (with the exception of $\operatorname{TMD}(3,2))$. Their dimensions are given in Table 1 .

Table 1.

Three infinite series of simple matroid designs that are not PMD's

$\begin{array}{ccccc}M & & r(M) & k(M) & \gamma(M) \\ \operatorname{TMD}(6 n+2,3) & n \geq 1 & 4 & 4 & 2(9 n+1) . \\ \operatorname{TMD}(6 n+4,3) & n \geq 0 & 4 & 4 & 2(9 n+4) \\ \operatorname{TMD}(q+1, q) & q>2 & 4 & q+1 & (q+1)(q-1) .\end{array}$

$q$ a prime power

For some values of $p$ and $q$ satisfying the conditions of (131), neither (i) nor (ii) is possible. $(p, q)=(3,17)$ is such a pair, for as may readily be verified, the consistency conditions of (132) do not hold for either of the designs $D_{3}(53,5,1)$ and $\operatorname{TD}_{3}(14,4,5,1)$. Hence every matroid design $M$ such that $\gamma(M)=51$ and $r(M) \geq 4$ is trivial.

Special constructions. The characterization theorems (124) and (131) excluded the cases $(p, q)=(2,3)$ and $(p, q)=(3,5)$. We shall therefore conclude by describing the possible rank 4 structures in these two cases. The methods of analysis are similar to those used in the preceding cases, and will be omitted here.

$\mathrm{EG}(3,2) \oplus P(2)$ and the inversive planes of order $3(a 3-(10,4,1)$ design $)$ are nontrivial members of $\Re_{4}(6)$. So also is the simple matroid design $\Psi$ defined above. The only other nontrivial member of $\Re_{4}(6)$ is the following construction.

Let $E^{\prime}$ be a set of 9 elements, and let $\mathcal{Q}_{i}=\left\{L_{1}^{i}, L_{2}^{i}, L_{3}^{i}\right\}$ for $i=1,2,3,4$, be the parallel classes of an $\operatorname{EG}(2,3)$ on the set $E^{\prime}$. We choose two "distinguished" parallel classes, say $\mathfrak{L}_{1}$ and $\mathfrak{L}_{2}$, and let $\mathcal{H}_{1}=\left\{L \cup L^{\prime}: L \in \mathfrak{L}_{1}\right.$ and $\left.L^{\prime} \in \mathfrak{I}_{2}\right\}$. Now let $a$ be a 2 -set disjoint from $E^{\prime}$, and let $\mathcal{H}_{2}=\left\{L \cup a: L \in \bigcup_{i=1}^{4} \varrho_{i}\right\}$. Then it is straightforward to check that $\Lambda=\left(E^{\prime} \cup a, \mathcal{H}_{1} \cup \mathcal{H}_{2}\right) \in \mathbb{M}_{4}(6)$. The points of $\Lambda$ are all simple except for $a$, and the lines of $\Lambda$ are all elementary except for the family of lines $\mathfrak{L}_{1} \cup \mathfrak{L}_{2}$.

Now let us consider $\prod_{4}(15)$. An argument like that used in the proof of (131) shows that the only nontrivial rank 4 matroid designs with cocircuit cardinality 15 are $D_{3}(17,5,1)$ (the unique inversive plane of order 4 ), a $\operatorname{TMD}(5,4)$, and the following special construction.

Let $E^{\prime}$ be a set of 16 elements, and let $\mathcal{L}_{i}=\left\{L_{1}^{i}, L_{2}^{i}, L_{3}^{i}, L_{4}^{i}\right\}$, for $i=1,2$, 
Table 2. List of matroid designs

\begin{tabular}{|c|c|c|}
\hline$\gamma$ & Rank 3 & Rank 4 \\
\hline 1 & - & - \\
\hline 2 & - & - \\
\hline 3 & - & - \\
\hline 4 & $\operatorname{PG}(2,2)$ & $\mathrm{EG}(3,2)$ \\
\hline 5 & - & - \\
\hline 6 & $\begin{array}{l}\mathrm{EG}(2,3) ; \mathrm{PG}(2,2) \oplus P(2) \\
\mathrm{EG}(2,2) \ominus \sigma(2,2,3)\end{array}$ & $\mathrm{EG}(3,2) \oplus P(2) ; \boldsymbol{\Psi} ; \mathrm{IP}(3) ; \boldsymbol{\Lambda}$ \\
\hline 7 & - & - \\
\hline 9 & $\operatorname{PG}(2,3)$ & - \\
\hline 10 & $D_{2}(13,3,1)$ & $D_{3}(14,4,1)$ \\
\hline 11 & - & - \\
\hline 13 & - & - \\
\hline 14 & $D_{2}(15,3,1) \oplus P(2)$ & $D_{3}(16,4,1) \oplus P(2) ; \operatorname{TMD}(6,3)$ \\
\hline 15 & $\begin{array}{l}\mathrm{EG}(2,4) \oplus P(3) \\
\mathrm{EG}(2,3) \oplus \sigma(3,2,4)\end{array}$ & $\mathrm{IP}(4) \oplus P(3) ; \operatorname{TMD}(5,4) ; \Omega$ \\
\hline 17 & - & - \\
\hline 19 & - & - \\
\hline 21 & $D_{2}(25,4,1)$. & $D_{3}(26,5,1) ?$ \\
\hline 22 & $D_{2}(25,3,1)$ & $D_{3}(26,4,1)$ \\
\hline 23 & - & - \\
\hline 25 & $P G(2,5)$ & - \\
\hline 26 & $D_{2}(27,3,1) \oplus P(2)$ & $D_{3}(28,4,1) \oplus P(2) ; \operatorname{TMD}(10,3)$ \\
\hline 29 & - & - \\
\hline 31 & - & - \\
\hline 33 & $D_{2}(37,4,1)$ & - \\
\hline 34 & $D_{2}(37,3,1)$ & $D_{3}(38,4,1)$ \\
\hline 35 & - & - \\
\hline 37 & $\cdot-$ & - \\
\hline 38 & $D_{2}(39,3,1) \oplus P(2)$ & $D_{3}(40,4,1) \oplus P(2) ; \operatorname{TMD}(14,3)$ \\
\hline 391 & $D_{2}(40,4,1) \oplus P(3)$ & $D_{3}(41,5,1) \oplus P(3) ? ; \operatorname{TMD}(11,4) ?$ \\
\hline 41 & - & - \\
\hline 43 & - & - \\
\hline 461 & $D_{2}(49,3,1)$ & $D_{3}(50,4,1)$ \\
\hline 47 & - & - \\
\hline 49 & $\operatorname{PG}(2,7)$ & - \\
\hline 51 & $D_{2}(52,4,1) \oplus P(3)$ & - \\
\hline 53 & - & - \\
\hline 551 & $D_{2}(61,6,1)$ & - \\
\hline 571 & $D_{2}(61,4,1)$ & $D_{3}(62,5,1) ?$ \\
\hline 581 & $D_{2}(61,3,1)$ & $D_{3}(62,4,1)$ \\
\hline 59 & - & - \\
\hline 61 & - & - \\
\hline
\end{tabular}



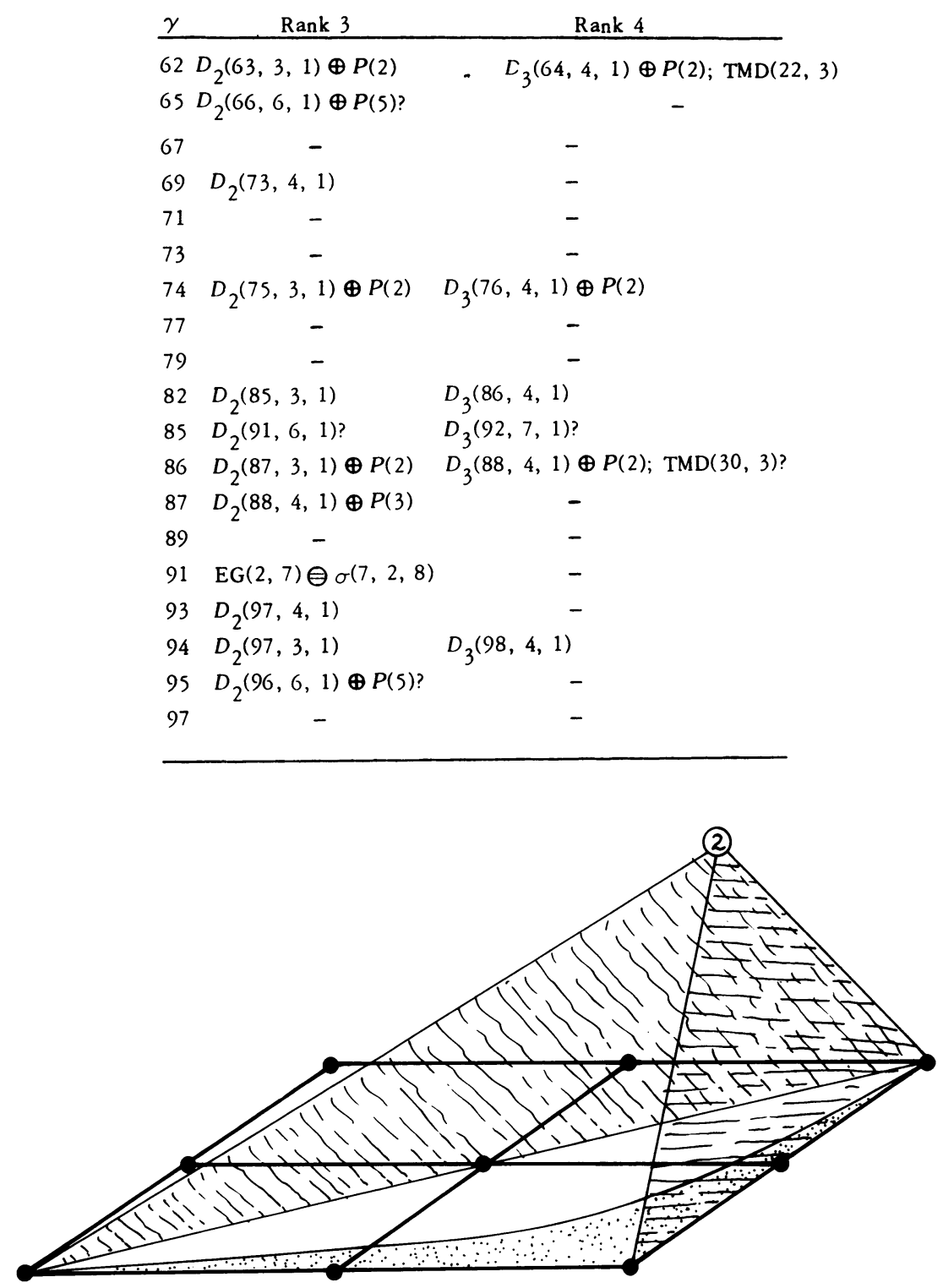

Figure 5. The matroid design $\Lambda$

$3,4,5$, be the parallel classes of an $\mathrm{EG}(2,4)$ on $E^{\prime}$. We choose two distinguished parallel classes, say $\mathfrak{L}_{1}$ and $\mathfrak{L}_{2}$, and let $\mathcal{H}_{1}=\left\{L \cup L^{\prime}: L \in \mathscr{L}_{1}\right.$ and $\left.L^{\prime} \in \mathfrak{I}_{2}\right\}$. Let $\mathcal{S}$ be the set of all 4 -subsets of $E^{\prime}$, no two members of which are contained together in a member of $\mathfrak{L}_{1} \cup \mathscr{L}_{2}$. It is easy to check that $|\mathfrak{S}|=4 \cdot 3 \cdot 2 \cdot 1=24$. Further, $\mathfrak{L}_{3} \cup \mathfrak{L}_{4} \cup \mathfrak{L}_{5} \subseteq \mathcal{S}$, and $\left|\mathfrak{I}_{3} \cup \mathfrak{I}_{4} \cup \mathfrak{I}_{5}\right|=12$. Letting $\mathcal{S}^{\prime}=\mathcal{S}-\left(\mathscr{L}_{3} \cup \mathfrak{L}_{4} \cup \mathfrak{L}_{5}\right)$, it follows that $\left|\mathcal{S}^{\prime}\right|=12$, and it is not difficult to see that $\mathcal{S}^{\prime}$ may also be partitioned into three parallel classes, say $\mathscr{L}_{3}^{\prime}, \mathscr{L}_{4}^{\prime}$, and $\mathfrak{L}_{5}^{\prime}$.

Now let $a$ and $b$ be two disjoint 3 -sets that are disjoint from $E^{\prime}$. Define 


$$
\begin{aligned}
& \mathcal{H}_{2}=\left\{L \cup a: L \in \mathcal{I}_{1} \cup \mathcal{I}_{2}\right\}, \quad \mathcal{H}_{3}=\{L \cup a: L \in \mathcal{S}\}, \\
& \mathcal{H}_{4}=\left\{L \cup b: L \in \mathcal{S}^{\prime}\right\}, \mathcal{H}_{5}=\left\{\{x\} \cup a \cup b: x \in E^{\prime}\right\} .
\end{aligned}
$$

It is then a straightforward matter to verify that $\left.\Omega=\left(E^{\prime} \cup a \cup b, \cup_{i=1}^{5} \mathcal{H}_{i}\right) \in\right)_{4}(15)$. The points of $\Omega$ are all simple, except for $a$ and $b$, and the lines of $\Omega$ are all elementary, except for the family of lines $\mathscr{\complement}_{1} \cup \mathfrak{\complement}_{2}$.

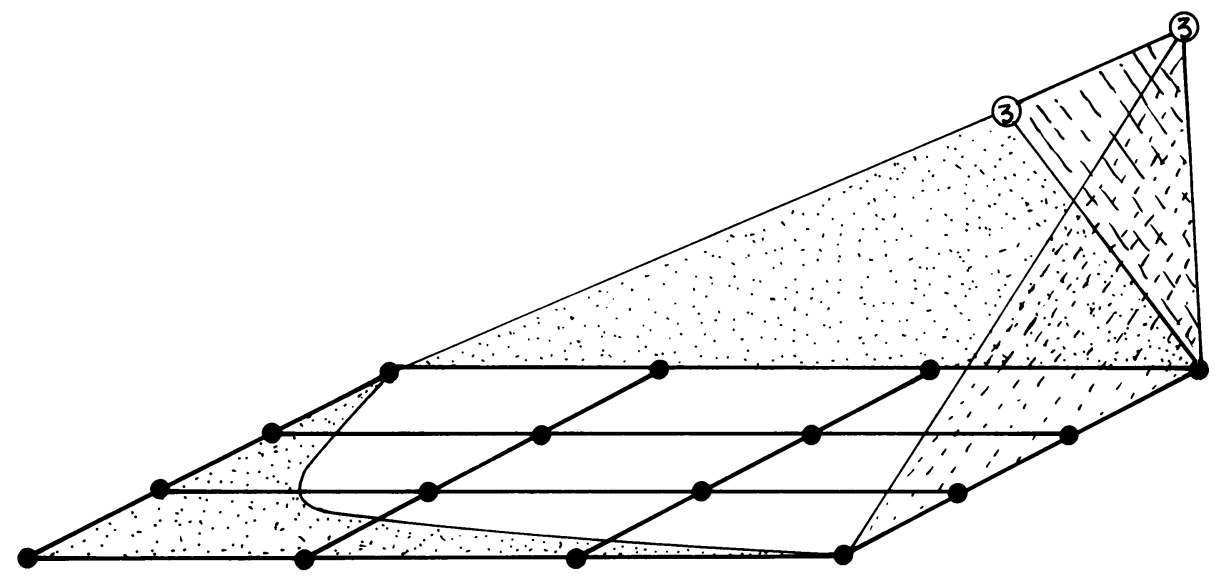

Figure 6. The matroid design $\Omega$

Concluding remarks. The preceding theorems define all matroid designs with cocircuit cardinalities $p$ a prime or prime squared. They further define all matroid designs of rank $\leq 4$ and cocircuit cardinality $p q$, where $p$ and $q$ are distinct primes such that $p<2 q / 3+1$. For certain values of $p$ and $q$, these theorems imply that $\mathbb{M}_{3}(p q)$ or $\mathbb{M}_{4}(p q)$ contains only trivioids, in which case $\mathbb{M}_{n}(p q)$ is known for every $n$. The characterization in the above cases is not complete only in the sense that the parameters for a $t$-design or a transversal design do not, in general, specify the structure of the $t$-design or TD uniquely.

In Table 2 we list all connected nontrivial matroid designs with cocircuit cardinality $\gamma$, where $1 \leq \gamma \leq 100$ and $\gamma$ is a prime or the product of two primes.

When $\gamma$ has three or more distinct prime factors, say $\gamma=p_{1} p_{2} \cdots p_{t}, t \geq 3$, the existence of a nontrivial $M \in \mathbb{M}_{n}\left(p_{i} p_{j}\right)$ implies the existence of a nontrivial $M^{\prime} \in \mathbb{M}_{n}(\gamma)$; namely, we take $M^{\prime}$ to be a $\gamma / p_{i} p_{j}$-inflation of $M$. However, it is certainly not in general true that every nontrivial member of $\mathbb{M}_{n}(\gamma)$ is an inflation of some nontrivial member of $\prod_{n}\left(p_{i} p_{j}\right)$, where $p_{i}$ and $p_{j}$ are prime factors of $\gamma$. For example, $\mathrm{EG}(2,4)$ is contained in $\mathbb{M}_{3}(12)$, but it is both simple and nontrivial.

The following is an interesting unsolved problem. For a given $\gamma$, is the rank of every nontrivial, connected, matroid design with cocircuit cardinality $\gamma$ bounded? The finiteness of Table 2 suggests that this is the case, although we have not even been able to establish the conjecture for $\gamma=6$. 
All matroid designs listed in Table 2 are known to exist, unless followed by a question mark. For existence theorems concerning $t$-designs the reader is referred to [6], [8], and [13]. IP $(s)$ denotes an inversive plane of order $s$.

\section{REFERENCES}

1. R. C. Bose and S. S. Shrikhande, On the construction of sets of mutually orthogonal latin squares and the falsity of a conjecture of Euler, Trans. Amer. Math. Soc. 95 (1960), 191-209. MR $22 \# 2557$.

2. K. A. Bush, Orthogonal arrays of index unity, Ann. Math. Statist. 23 (1952), 426434. MR 14, 125 .

3. H. Crapo and G. C. Rota, On the foundations of combinatorial theory: Combinatorial geometries, M.I.T., Cambridge, Mass., 1970.

4. P. Dembowski and A. Wagner, Some characterizations of finite projective spaces, Arch. Math. 11 (1960), 465-469. MR $26 \# 660$.

5. M. Hall, Jr., Combinatorial theory, Blaisdell, Waltham, Mass., 1967. MR 37 \#80.

6. H. Hanani, On quadruple systems, Canad. J. Math. 12 (1960), 145-147. MR 22 \#2558.

7. - The existence and construction of balanced incomplete block designs, Ann. Math. Statist. 32 (1961), 361-386. MR 29\#4161.

8. - On some tactical configurations, Canad. J. Math. 15 (1963), 702-722. MR $28 \# 1136$.

9. W. Kantor, Characterizations of finite projective and affine spaces, Canad. J. Math. 21 (1969), 64-75. MR $38 \# 4338$.

10. U. S. R. Murty, Equicardinal matroids and finite geometries, Calgary Internat. Conf. Combinatorial Structures and their Applications, Gordon and Breach, New York, 1969.

11. W. T. Tutte, Lectures on matroids, J. Res. Nat. Bur. Standards Sec. B 69B (1965), 1-47. MR 31 \#4023.

12. H. Whitney, On the abstract properties of linear dependence, Amer. J. Math. 57 (1935), 509-533.

13. E. Witt, Über Steinersche Systeme, Abh. Math. Sem. Univ. Hamburg 12 (1938), $265-275$.

14. H. P. Young, U. S. R. Murty and J. Edmonds, Equicardinal matroids and matroid designs, Proc. Second Annual Chapel Hill Conf. on Combinatorial Math. and Appl., Chapel Hill, N. C., 1970.

15. H. P. Young and J. Edmonds, Matroid designs, J. Res. Nat. Bur. Standards Sect. B $77 B$ (1973).

DEPARTMENT OF MATHEMATICS, GRADUATE CENTER, CITY UNIVERSITY OF NEW YORK, NEW YORK, NEW YORK 10036 